\title{
Bifurcation to Infinitely Many Sinks
}

\author{
Clark Robinson` \\ Mathematics Department, Northwestern University, Evanston, IL 60201, USA
}

\begin{abstract}
This paper considers one parameter families of diffeomorphisms $\left\{F_{t}\right\}$ in two dimensions which have a curve of dissipative saddle periodic points $P_{t}$, i.e. $F_{t}^{n}\left(P_{t}\right)=P_{t}$ and $\left|\operatorname{det} D F_{t}^{n}\left(P_{t}\right)\right|<1$. The family is also assumed to create new homoclinic intersections of the stable and unstable manifolds of $P_{t}$ as the parameter varies through $t_{0}$. Gavrilov and Silnikov proved that if the new homoclinic intersections are created nondegenerately at $t_{0}$, then there is an infinite cascade of periodic sinks, i.e. there are parameter values $t_{n}$ accumulating at $t_{0}$ for which there is a sink of period $n$ [GS2, Sect. 4]. We show that this result is true for real analytic diffeomorphisms even if the homoclinic intersection is created degenerately. We give computer evidence to show that this latter result is probably applicable to the Hénon map for $A$ near 1.392 and $B$ equal -0.3 .

Newhouse proved a related result which showed the existence of infinitely many periodic sinks for a single diffeomorphism which is a perturbation of a diffeomorphism with a nondegenerate homoclinic tangency. We give the main geometric ideas of the proof of this theorem. We also give a variation of a key lemma to show that the result is true for a fixed one parameter family which creates a nondegenerate tangency. Thus under the nondegeneracy assumption, not only is there a cascade of sinks proved by Gavrilov and Silnikov, but also a single parameter value $t^{*}$ with infinitely many sinks.
\end{abstract}

\section{Introduction}

The existence of a cascade of sinks is important because it analyzes a sequence of bifurcation which is different than period doubling. The existence of infinitely many sinks in Theorem $\mathrm{C}$ shows that there are generic situations which often arise where points tend to infinitely many distinct attractors. It indicates that for certain parameter values near $A=1.392$ the Hénon map does not have a transitive strange attractor but actually many different periodic sinks with narrow basins of attraction. (See Example 2.4 below.)

* Partially supported by National Science Foundation Grant MSC 80-02177 
These results for diffeomorphisms have similarities with bifurcations of maps of the intervals and also differences. A family of maps of the interval $\left\{f_{t}\right\}$ nondegenerately creates homoclinic intersections if there is an unstable periodic point $P_{t}$ and a nondegenerate critical point $Q_{t}$ in the unstable manifold of $P_{t}$, such that $Q_{t}$ is mapped back to $P_{t}$ by some power of $f_{t}\left(Q_{t}\right.$ is an eventually periodic critical point). If the family $\left\{f_{t}\right\}$ nondegenerately creates homoclinic intersections, then there is an infinite cascade of sinks as in Theorem A below, but there is no one parameter value with infinitely many sinks as in Theorem C. See Remark 8.3 below and [V] for further discussion of this comparison.

Newhouse originally proved there exists a specific example where there is a residual subset $\mathscr{R}$ of an open set of diffeomorphisms $\mathscr{N}$ such that each $G$ in $\mathscr{R}$ has infinitely many sinks, [N2]. Later he proved this occurred near any dissipative diffeomorphism $F$ which had a nondegenerate tangency of stable and unstable manifolds, [N4]. This later result follows from first proving that there is an open set of diffeomorphism in the $C^{2}$ topology $\mathscr{N}$, which is $C^{2}$ near $F$ such that for each $G$ in $\mathscr{N}$ there is a hyperbolic basic set $\Lambda=\Lambda(G)$ which has $W^{u}(\Lambda)$ having a nondegenerate tangency with $W^{s}(\Lambda)$. He also proved, [N4, Theorem 3], that for a fixed one parameter family of diffeomorphisms $\left\{F_{t}\right\}$ which creates a nondegenerate tangency of stable and unstable manifolds of a $n$ periodic point $P_{t}$ at $t=t_{0}$, where $\left|\operatorname{det} D F^{n}\left(P_{t}\right)\right|<1$, then for any $\varepsilon>0$ there is an interval $\left[t_{1}, t_{2}\right]$ $C\left[t_{0}-\varepsilon, t_{0}+\varepsilon\right]$ such that for $t$ in $\left[t_{1}, t_{2}\right] F_{t}$ has a nondegenerate tangency of the stable and unstable manifolds of a hyperbolic basic set $\Lambda_{t}$ containing $P_{t}$. He has stated in talks and implies in [N4, Remark 1, p. 105], but does not state explicitly in his papers, that there is a residual subset $J$ in $\left[t_{1}, t_{2}\right]$ such that for $t$ in $J$ the fixed one parameter family $F_{t}$ has infinitely many sinks, [N4, Remark 1, p. 105]. Theorem E below states and proves that this is indeed true. See also [N5, Theorem 8.1], and [GH]. Earlier Garilov and Silnikov had proved that if a $C^{3}$ family nondegenerately creates the homoclinic intersection then there is an infinite cascade of sinks as in Theorem A below, [GS2, Sect. 4]. They also showed that hyperbolic invariant sets were created which are not conjugate to each other, so the system is inaccessible by a simple bifurcation from at least one side, [GS] or [GH].

This paper proves a key result, Proposition 3.3, that whenever there is a one parameter family $\left\{F_{t}\right\}$ and a box $B$ such that the images of the box $F_{t}(B)$ are pulled across $B$ in the shape of a horseshoe, then there is an interval of parameter values $J$ such that for $t$ in $J, F_{t}$ has at least one periodic sink. Using this result it can be shown that a fixed one parameter family of real analytic diffeomorphism which creates homoclinic intersections (as defined in Sect. 2) has an infinite cascade of periodic sinks, Theorem A. Therefore there is a sequence of periodic sinks $p_{n}$ for different parameter values $t_{n}$, but not necessarily infinitely many sinks for one parameter value $t^{*}$. This theorem has fairly weak hypothesis. It is almost certainly applicable to the Hénon map to show there is an infinite cascade of sinks for $B=-0.3$ and $A$ near 1.39. See Example 2.4. Using the stronger hypothesis used in [N4] that the family nondegenerately creates homoclinic intersections, Proposition 3.3 is the only new ingredient needed to show that the one parameter family can be fixed and prove there are many parameter values which have infinitely many sinks, Theorem C. Most of the work goes into proving that if $\left\{F_{t}\right\}$ 
nondegenerately creates homoclinic intersections, then there is a persistent tangency of stable and unstable manifolds, Theorem D. This result is proved in [N4]. Since its proof is very involved, we present the main aspects of the proof with reference for the analytic details. (This result is stated in [N5] but those lectures do not discuss the proof.)

The outline of the paper is as follows. Section 2 states the main results and gives examples where the theorem applies. Section 3 states and proves the proposition on the creation of one sink. Section 4 proves that if a family of real analytic diffeomorphisms create intersections then they create odd order intersections. Section 5 proves the result on the existence of an infinite cascade of sinks. Section 6 discusses the persistence of intersection of "thick" Cantor sets. Section 7 shows how thick Cantor sets of stable manifolds arise from the nondegenerate creation of homoclinic tangency. Section 8 shows how the parameter value can be chosen to get both a thick Cantor set of stable manifolds and a nondegenerate tangency - hence a persistent tangency. Finally Sect. 9 proves that the persistence of nondegenerate tangency leads to infinitely many sinks.

\section{Statement of Main Theorem and Applications}

For a diffeomorphism $F$ let $D F(p)$ denote the derivative of $F$ at $p$, i.e. the matrix of partial derivatives. A point $p$ is called a periodic sink (respectively source) for a diffeomorphism $F$ if $p$ is a periodic point, $F^{n}(p)=p$, such that all eigenvalues of the derivative of $F^{n}, D F^{n}(p)$, have absolute value less than one (respectively all have absolute value greater than one). Thus a sink is a periodic attractor and there is a neighborhood $U$ of the orbit $\mathcal{O}(p)=\left\{F^{j}(p): j\right.$ in $\left.Z\right\}$ such that $\bigcap_{j \geqq 0} F^{j}(U)=\mathcal{O}(p)$. A point $p$ is called a periodic saddle point for a diffeomorphism $F$ in two dimensions if it is a periodic point with $F^{n}(p)=p$ for some $n$ and the eigenvalues of $D F^{n}(p)$ are $\lambda_{u}$ and $\lambda_{s}$, both real, with $\left|\lambda_{u}\right|>1$ and $\left|\lambda_{s}\right|<1$. The stable and unstable manifolds of a saddle point for a $C^{r}$ diffeomorphism $F$ are then $C^{r}$ curves tangent to the eigendirections defined by $W^{s}(p, F)=\left\{q\right.$ : distance $F^{j}(q)$ to $F^{j}(p)$ goes to zero as $j$ goes to infinity $\}$ and $W^{u}(p, F)=\left\{q\right.$ : distance $F^{j}(q)$ to $F^{j}(p)$ goes to zero as $j$ goes to minus infinity\}.

We need to distinguish the direction of crossing of two oriented curves and the order of tangency. Let $\gamma^{1}$ and $\gamma^{2}$ be two oriented differentiable curves. We say that $\gamma^{1}$ has positive (respectively negative) intersection with $\gamma^{2}$ at a point $p$ if $p$ is on both curves and there are local coordinates $(x, y)$ near $p$ with $x(p)=y(p)=0$ and in which there are oriented parametrizations $\left\{\left(x_{j}(s), y_{j}(s)\right):|s|<\varepsilon\right\}$ of $\gamma^{j}$ with (i) $y_{2}(s) \equiv 0$, $x_{2}(0)=0$, and $x_{2}^{\prime}(0)>0$ and (ii) $y_{1}(0)=0=x_{1}(0), y_{1}(s)<0$ for $-\varepsilon<s<0$ (respectively $0<s<\varepsilon$ ), and $y_{1}(s)>0$ for $0<s<\varepsilon$ (respectively $-\varepsilon<s<0$ ). We say that $\left\{\gamma^{1}, \gamma^{2}\right\}$ have intersection of order $n+1$ at $p$ (or tangency of order $n$ ) if there are parametrizations as above with (i) $y_{2}(s) \equiv 0$ and (ii) $y_{1}(0), y_{1}^{\prime}(0)=0, \ldots, y_{1}^{(n)}(0)=0$, but $y^{(n+1)}(0) \neq 0$. Note that $\gamma^{1}$ and $\gamma^{2}$ are transverse at $p$ if and only if they have intersection of order one if only if they have tangency of order zero.

We say that $F_{t}$ creates (respectively destroys) homoclinic intersections at $t_{0}$ for a periodic saddle point $P_{t}$ if there are $\varepsilon>0, Q_{t}=F_{t}^{k}\left(P_{t}\right)$ for some $k$, and continuously 
varying subarcs $\gamma_{t}^{s} \subset W^{s}\left(P_{t}, F_{t}\right)$ and $\gamma_{t}^{u} \subset W^{u}\left(Q_{t}, F_{t}\right)$ for $t_{0}-\varepsilon \leqq t \leqq t_{0}+\varepsilon$ such that

(i) $\gamma_{t}^{s} \cap \gamma_{t}^{u}=\emptyset$ for $t_{0}-\varepsilon \leqq t<t_{0}$ (respectively, $t_{0}<t \leqq t_{0}+\varepsilon$ ),

(ii) for $t_{0}<t \leqq t_{0}+\varepsilon$ (respectively, $t_{0}-\varepsilon \leqq t<t_{0}$ ) $\gamma_{1}^{s}$ has both positive and negative intersections with $\gamma_{t}^{u}$.

We say that $\left\{F_{t}\right\}$ creates odd order homoclinic intersections at $t_{0}$ if condition (i) is satisfied and

(ii) for $t_{0}<t \leqq t_{0}+\varepsilon \gamma_{t}^{u}$ has at least one positive intersection with $\gamma_{t}^{\mathrm{s}}$ of odd order and at least one negative intersection with $\gamma_{t}^{s}$ of odd order.

In condition (ii)' there can be more than two intersections some of which are of even order. In the proof of Theorem A below show that if $\left\{F_{t}\right\}$ is a family of analytic diffeomorphisms which depend continuously on $t$ and satisfying (ii) then it satisfies condition (ii). Also in the proof of Theorem B condition (ii) is not needed for all $t_{0}<t \leqq t_{0}+\varepsilon$ but only for a sequence of parameter values $t_{j}>t_{0}$ which accumulate on $t_{0}$. The definition of (ii)' could be weakened accordingly.

The above condition is sufficient to prove there is a cascade of sinks, but to prove that there are infinitely many sinks for a single parameter value another condition is needed. We say that $\left\{F_{t}\right\}$ nondegenerately creates homoclinic intersections at $t_{0}$ if $\left\{F_{t}\right\}$ creates odd order homoclinic intersections [conditions (i) and (ii)'] and

(iii) $\gamma_{t_{0}}^{s}$ and $\gamma_{t_{0}}^{u}$ have intersection of order two (tangency of order one),

(iv) If coordinates are taken so $\gamma_{t}^{s}$ lies on $y=0$ and $y^{*}(t)$ is the extreme value of $y$ along $\gamma_{t}^{u}$, then $d y^{*} / d t \neq 0$ at $t=t_{0}$.

Note in this case, the intersections for $t>t_{0}$ are necessarily transverse, i.e. of order one. If $\left\{F_{t}\right\}$ nondegenerately creates homoclinic intersections, Newhouse uses the terminology that it creates a nondegenerate tangency at $t_{0}$. His terminology emphasizes the tangency in condition (iii), while ours emphasizes the topologically transverse intersections in condition (ii).

Theorem A. Let $\left\{F_{t}\right\}$ be a one parameter family of real analytic diffeomorphisms in two dimensions which depend continuously on t. Assume it creates (or destroys) a homoclinic intersection at $t_{0}$ for the periodic points $P_{t}$ of periodic $n$ with $\left|\operatorname{det} D F_{t_{0}}^{n}\left(P_{t_{0}}\right)\right|<1$. Then $F_{t}$ has an infinite cascade of sinks. More specifically there is a sequence of parameters values $t_{j}$ converging monotonically to $t_{0}$ such that $F_{t_{j}}$ has a periodic sink of period $n_{j}$. The orbits of the sinks pass near the point of tangency of $W^{s}\left(P_{t_{0}}, F_{t_{0}}\right)$ and $W^{u}\left(F^{k}\left(P_{t_{0}}\right), F_{t_{0}}\right)$. The periods $n_{j}$ of the sinks grow like $n_{j+1}-n_{j}=n$ or $2 n$ depending on whether $F_{t}$ preserves the orientations on $W^{s}\left(P_{t}, F_{t}\right)$ and $W^{u}\left(P_{t}, F_{t}\right)$ or not.

The result for real analytic diffeomorphisms follows quite directly from the following result about $C^{j}$ diffeomorphisms.

Theorem B. Assume $\left\{F_{t}\right\}$ is a one parameter family of $C^{j}$ diffeomorphisms in Theorem A but assume it creates (or destroys) odd order homoclinic intersections at $t_{0}$ of oder $j$. Then the conclusion of Theorem $A$ is true. Here $j \geqq 1$.

2.1. Remark. Curry and Johnson, [CJ], calculated by means of a computer the asymptotic rate of the creation of sinks in the cascade for the family of maps studied in [ACHM]. They noted that

$$
\lim _{n \rightarrow \infty}\left(t_{n}-t_{n-1}\right) /\left(t_{n+1}-t_{n}\right)=\lambda,
$$


where $\lambda_{u}$ is the unstable eigenvalue of the saddle fixed point. They also include a proof with details to be supplied elsewhere. As noted above Gavrilov and Silnikov proved Theorem A under the assumption that a $C^{3}$ family nondegenerately creates homoclinic intersections. They also proved bounds on the parameter values for the existence of the sink of period $n$ which imply the asymptotic rate of creation of sinks noted by Curry and Johnson, [GS2, 4.4 and 4.5]. At the end of Sect. 5, we indicate how this asymptotic rate is related to the proof of Theorem B.

The following theorems are essentially the results of Newhouse. In particular Theorem D is [N2, Theorem 2].

Theorem C. Suppose $\left\{F_{t}\right\}$ is a fixed one parameter family of $C^{3}$ diffeomorphisms of a two manifold which nondegenerately creates homoclinic intersections at $t_{0}$ for the periodic points $P_{t}$ of period $n$ with $\left|\operatorname{det} D F_{t_{0}}^{n}\left(P_{t_{0}}\right)\right|<1\left(\right.$ respectively $\left.\left|\operatorname{det} D F_{t_{0}}^{n}\left(P_{t_{0}}\right)\right|>1\right)$. Then given $\varepsilon>0$ there is a subinterval $\left[t_{1}, t_{2}\right] \subset\left[t_{0}-\varepsilon, t_{0}+\varepsilon\right]$ and a residual subset $J \subset\left[t_{1}, t_{2}\right]$ such that for $t$ in $J, F_{t}$ has infinitely many sinks (respectively sources).

In Theorem $C$ we assume $\left\{F_{t}\right\}$ is a $C^{1}$ curve of $C^{3}$ diffeomorphisms, i.e. the third derivative of $F$ with respect to $q$ in $M$ has one continuous derivative with respect to the parameter $t . F_{t}$ is assumed $C^{3}$ in order to almost $C^{2}$ linearize near $P_{t}$. See Sect. 7 for more details.

2.2. Remark. Theorem $\mathrm{C}$ can not be proved by showing the intervals of parameter values with sinks given in Theorem A overlap. In fact, Remark 5.2 indicates why no two of the sinks of Theorem A occur for the same parameter value. In terms of bifurcation subsets of the function space of diffeomorphisms, Theorem $\mathrm{C}$, or more precisely [N4, Theorem 1], means that there is an open set of $C^{2}$ diffeomorphisms, $\mathscr{N}$, such that $\Sigma_{1}=\{G \in \mathscr{N}: G$ has a generic saddle node $\}$ is dense in $\mathscr{N}$. Such bifurcations are codimension one, for each $G$ in $\Sigma_{1}$ there is a codimension one submanifold $\Sigma_{1}(G)$ in $\mathscr{N}$ such that each $H$ in $\Sigma_{1}(G)$ has a saddle node bifurcation. A generic arc crosses these bifurcations transversally. Theorem $\mathrm{E}$ does not prove there are transverse crossings but does prove there are infinitely many sinks. If the periodic point $P_{t}$ has eigenvalues that are independent enough to $C^{2}$ linearize near $P_{t}$, then it appears that the bifurcations are actually generic saddle node bifurcations. This result would use a lemma like [N2, Lemma 2] and is not included in this paper.

Theorem $\mathrm{C}$ follows from Theorems $\mathrm{D}$ and $\mathrm{E}$ below. To state these results we need further definitions. See [N5] for more precise statements and examples. The creation of homoclinic intersections implies the creation of Smale horseshoes. In fact there are integers $n$, boxes $B_{n}$, and parameter values $t=t_{n}$ such that $F_{t}^{n}\left(B_{n}\right)$ crosses $B_{n}$ in the shape of a horseshoe, see Fig. 5b below. Letting $G=F_{t}^{n}$ and $B=B_{n}$, the set $A=\left\{G^{k}(B): k\right.$ is in $\left.Z\right\}$ is the maximal invariant set for $G$ in $B$, i.e. $A$ is the set of all points $q$ such that both the forward and backward orbit of $q$ by $G$ stays in $B$. Each point $q$ in $\Lambda$ also has a contracting (stable) direction $E_{q}^{s}$ and an expanding (unstable) direction $E_{q}^{u}$ much like the eigendirections at a saddle fixed point. More precisely, a closed invariant set $\Lambda$ for $G$ is said to have a hyperbolic structure if the tangent space of the ambient manifolds has a splitting at points $q$ of $\Lambda, T_{q} M=E_{q}^{s}+E_{q}^{u}$, where the splitting varies continuously with $q$, and if there are constants $C>0$ and $\lambda>1$ such that for $k \geqq 0$ and for $v^{s}$ in $E_{q}^{s},\left|D G^{k}(q) v^{s}\right| \leqq C \lambda^{-k}\left|v^{s}\right|$, and for $v^{u}$ in $E_{q}^{u},\left|D G^{-k}(q) v^{u}\right| \leqq C \lambda^{-k}\left|v^{u}\right|$. If $\Lambda$ has a hyperbolic structure for $G$, then 
the nonlinear map $G$ has a family of invariant nonlinear manifolds tangent to the linear directions $E_{q}^{s}$ and $E_{q}^{u}$ which are contracted and expanded respectively by $G$. More precisely, for each point $q$ in $\Lambda$, the stable manifold of $q$ for $G$ is the set $W^{s}(q, G)=\left\{m\right.$ : distance $G^{k}(q)$ to $G^{k}(m)$ goes to zero as $k$ goes to infinity $\}$. The local stable manifold of $q$ of size $\varepsilon>0$ is the set $W_{\varepsilon}^{s}(q, G)=\left\{m\right.$ in $W^{s}(q, G)$ : distance $G^{k}(q)$ to $G^{k}(m)$ is less than $\varepsilon$, for all $\left.k \geqq 0\right\}$. Thus $W^{s}(q, G)=\bigcup\left\{G^{-j} W_{\varepsilon}^{s}\left(G^{j}(q), G\right): j \geqq 0\right\}$. If $\Lambda$ has a hyperbolic structure for a $C^{r}$ diffeomorphism $G$, then (i) $W_{\varepsilon}^{s}(q, G)$ is a $C^{r}$ differentiable disk with dimension equal to $\operatorname{dim} E_{q}^{s}$, (ii) the $\operatorname{disks} W_{\varepsilon}^{s}(q, G)$ vary continuously in the $C^{r}$ topology as $q$ varies in $A$, and (iii) the disks are invariant, $G\left(W_{i}^{s}(q, G)\right) \subset W_{i}^{s}(G(q), G)$. The (global) stable manifold is an immersed $C^{r}$ differentiable manifold. Similarly the unstable manifold of $q$ for $G$ is the set $W^{u}(q, G)$ $=\left\{m\right.$ : distance $G^{k}(q)$ to $G^{k}(m)$ goes to zero as $k$ goes to minus infinity $\}$ and the local unstable manifold of size $\varepsilon$ is $W_{\varepsilon}^{u}(q, G)=\left\{m\right.$ in $W^{u}(q, G)$ : distance $G^{k}(q)$ to $G^{k}(m)$ is less than $\varepsilon$ for all $k \leqq 0\}$. Again $W^{u}(q, G)=\bigcup\left\{G^{j}\left(W_{\varepsilon}^{u}\left(G^{-j}(q), G\right)\right): j \geqq 0\right\}$. A closed set $\Lambda$ is called a hyperbolic basic set for $G$ if (i) it is invariant for $G, G(A)=A$, (ii) it has a hyperbolic structure for $G$, (iii) there is a point $q$ in $A$ with a dense orbit, $\operatorname{closure}(\mathcal{O}(q))=\Lambda$, and (iv) $\Lambda$ has a local product structure, i.e. if $\varepsilon>0$ is sufficiently small and $p, q$ are in $\Lambda$ then $W_{\varepsilon}^{u}(p, G) \cap W_{\varepsilon}^{s}(q, G) \subset \Lambda$.

If $\Lambda$ is a hyperbolic basic set then there is a neighborhood $U$ of $\Lambda$ such that $\bigcap_{j=-\infty}^{\infty} F^{j}(U)=\Lambda$. For $G$ which is $C^{1}$ near $F, \Lambda(G)=\bigcap_{j} G^{j}(U)$ is a hyperbolic basic set for $G$. A hyperbolic basic set $\Lambda$ is called a wild hyperbolic set for $F$ (or has persistent tangencies of stable and unstable manifolds) if for any $G$ which is $C^{2}$ near $F$ there are points $q_{1}$ and $q_{2}$ in $\Lambda(G)$ for which $W^{s}\left(q_{2}, G\right)$ has a nondegenerate tangency with $W^{u}\left(q_{1}, G\right)$. (See [N5] for further definitions and more precise statements.)

Theorem $\mathrm{C}$ follows from the following two theorems.

Theorem D. Suppose $\left\{F_{t}\right\}$ nondegenerately creates homoclinic intersections at $t_{0}$ for the curve of periodic points $P_{t}$ with $\left|\operatorname{det} D F_{t_{0}}^{n}\left(P_{t_{0}}\right)\right| \neq 1$ and each $F_{t}$ is $C^{3}$. Then given $\varepsilon>0$ there is a subinterval $\left[t_{1}, t_{2}\right] \subset\left[t_{0}-\varepsilon, t_{0}+\varepsilon\right]$ such that for $t$ in $\left[t_{1}, t_{2}\right], F_{t}$ has a wild hyperbolic set containing the corresponding periodic point $P_{t}$ of period $n$.

Theorem E. Assume $\left\{F_{t}\right\}$ has an interval of parameter values $\left[t_{1}, t_{2}\right]$ such that for $t$ in $\left[t_{1}, t_{2}\right], F_{t}$ has a wild hyperbolic set containing a periodic point $P_{t}$ with $\left|\operatorname{det} D F_{t}^{n}\left(P_{t}\right)\right|<1$ (respectively $>1$ ). Then there is a residual subset $J \subset\left[t_{1}, t_{2}\right]$ such that for $t$ in $J, F_{t}$ has infinitely many sinks (respectively sources).

2.3. Remark. If $F_{t}$ are area preserving then Theorem D is unknown. It can be shown that $F_{t}$ goes through a cascade of bifurcations producing elliptic points as in Sect. 5 below. See [N3].

2.4. Example. Recently there has been much interest in the Hénon map

$$
F_{A B}(x, y)=\left(A-B y-x^{2}, x\right) \text {. }
$$

For $B=0$, the map is like the graph of a parabola map of the interval. For certain values of $A$ the end of the parabola gets mapped to the line $x=x_{0}$ containing one of the fixed points. This line is in the stable manifold of the fixed point. Thus as $A$ 


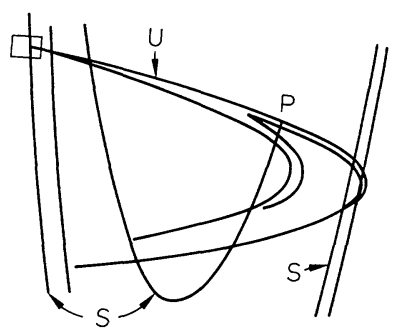

a)

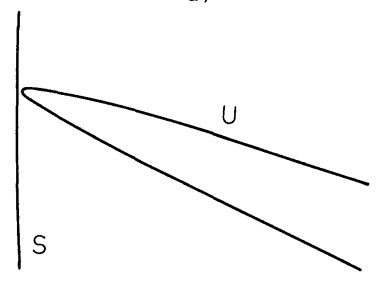

c)

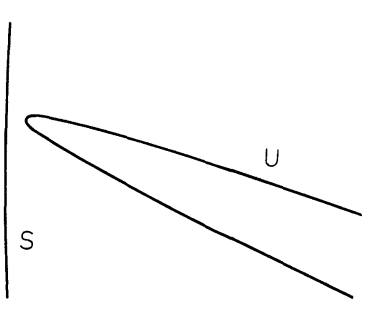

b)

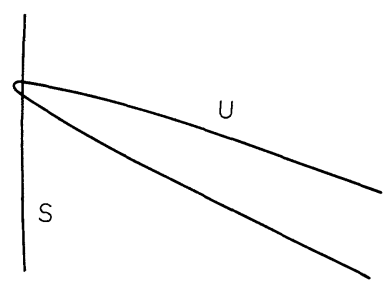

d)

Fig. 1a-d. Hénon map. The stable and unstable manifolds of the fixed point $P$ are labelled by $S$ and $U$, respectively. The small box in $\mathbf{a}$ is enlarged in $\mathbf{b}-\mathbf{d}$ for different values of $A . B=-0.3$. a $A=1.39$, b $A=1.39$, c $A=1.392, \mathbf{d} A=1.395$

varies it creates a nondegenerate tangency. This holds for $|B|$ small enough. Therefore for small $B$ there are values of $A$ with infinitely many sinks. See $[\mathrm{V}$, Theorem $\mathrm{D}]$ for details.

Earlier, [N4, Remark 1, p. 105] had indicated that for some parameter values the Hénon map has infinitely many sinks. It does not specify the values of $A$ and $B$ for which this is true, but oral communication indicated it is for this case with $|B|$ small.

More interestingly, for $B=-0.3$ and as $A$ varies from 1.39 to 1.4 , computer studies indicate $F_{A}$ creates homoclinic intersections. See Fig. 1. This fact is probably verifiable either via more careful computer studies or analytically. Because everything is analytic, if $\left\{F_{A}\right\}$ does create homoclinic intersections then there is an infinite cascade of sinks. Therefore the computer studies strongly indicate that there is an infinite cascade of sinks.

Further the homoclinic tangency appears nondegenerate in the computer studies: the stable manifold has small curvature while the curvature of the unstable manifold is very large (it appears as a sharp point). See Fig. 1. Therefore it is indicated, but is unproved analytically or via computers, that there are values of $A$ between 1.39 and 1.4 for $B=-0.3$ with infinitely many sinks. The nondegeneracy condition appears much more difficult to prove than the creation of homoclinic intersections.

The plots of iterates of a single point for $A$ between 1.39 and 1.4 and $B=-0.3$ all appear like attractors. One aspect of the explanation of why the sinks are not visible is that the basis of attraction of the sinks are very narrow. Further theoretical and numerical explanation is needed. 


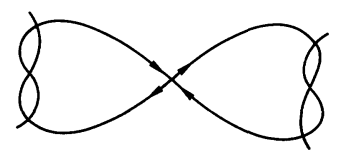

a)

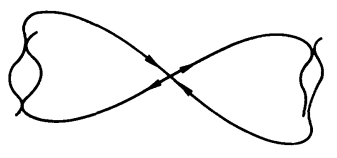

b)

Fig. 2a and b. Forced Duffing equation with damping. a $\delta=0, \varepsilon>0 ; \mathbf{b} \delta=\delta_{0}>0, \varepsilon>0$

These computer results contrast with the result of Misiurewicz, [Mis], for the Lozi map for which a hyperbolic attractor exists. The difference is that the smooth bend in the Hénon map causes a saddle node to be created as the image of the box is pulled across itself. The "piecewise linear" character of the Lozi map avoids this and immediately creates two saddle points.

The paper by Aronson et al. [ACHM], contains computer studies of an equation which models delayed regulation of population growth. Their studies like those for the Hénon map indicate the creation of odd order homoclinic intersections.

2.5. Example. Another type of example that has been studied is forced oscillators. Consider the forced Duffing equation with damping

$$
\begin{aligned}
& \dot{x}=v, \\
& \dot{v}=\beta x-\alpha x^{3}+\varepsilon(\gamma \cos \omega t-\delta v) .
\end{aligned}
$$

For $\delta=0$, and $0<\varepsilon<\varepsilon_{0}$ there is a transverse homoclinic orbit for the time $2 \pi$ map. This can be seen by using the Melnikov integral, [HM, Sect. 4]. As $\delta$ increases, the dissipation pulls the unstable manifold inward creating a nondegenerate tangency at $\delta=\delta_{0}$. (This corresponds to $\gamma=\gamma_{0}$ in [HM].) Thus for $\delta$ near $\delta_{0}$ there are infinitely many sinks.

2.6. Example. Levi considered a forced van der Pol equation of the type studied by Levinson

$$
\varepsilon \ddot{x}+\phi(x) \dot{x}+\varepsilon x=b p(t)
$$

where $\phi(x)$ and $p(t)$ are rounded off square periodic functions. He showed there were hyperbolic basic sets for certain values of $b$. Moreover he showed for other values of $b$, there are nondegenerate homoclinic tangency and so infinitely many sinks [L, p. 33 and Sect. 3.6].

\section{Creation of One Sink}

3.1. Example. Before considering the creation of sinks in two dimensions, consider a map of the real line $f_{t}: \mathbb{R} \rightarrow \mathbb{R}$ with $\partial^{2} f_{t} / \partial x^{2} \geqq a>0, \partial f_{t} / \partial t<0$; and $f_{0}(x)>x$, e.g. $f_{t}(x)=x^{2}-t+2$. As $t$ increases there is a first parameter value $t_{1}$ where $f_{t_{1}}\left(x_{1}\right)=x_{1}$. At this point $f^{\prime}\left(x_{1}\right)=1$.

Then for $t_{1}<t<t_{1}+\varepsilon, f_{t}(x)=x$ has two solutions $x_{t}<x_{t}^{\prime}$, and $0<f_{t}^{\prime}\left(x_{t}\right)<1$ $<f_{t}^{\prime}\left(x_{t}^{\prime}\right)$. Thus $x_{t}$ is a sink for $f_{t}$ (and $x_{t}^{\prime}$ is a source). 


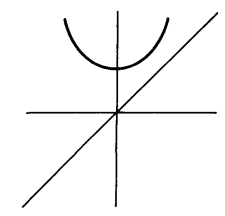

a)

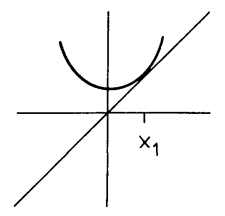

b)

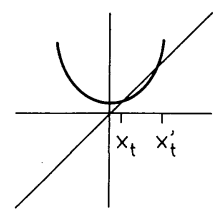

c)

Fig. 3a-c. Graph of $y=f(x)$ with diagonal $y=x$. (a) $t<t_{1} ;$ (b) $t=t_{1} ;$ (c) $t>t_{1}$

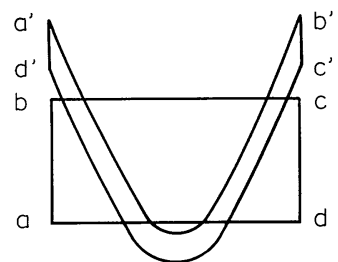

Fig. 4. The box $-2 \leqq x \leqq 2,-2 \leqq y \leqq 2$ with vertices $a, b, c, d$. The primed letters label the vertices which are the images of the vertices labelled by the corresponding unprimed letters, e.g., $a^{\prime}=F_{A}(a)$

\subsection{Example. Next consider a Hénon type map}

$$
F_{A}(x, y)=\left(y,-A-B x+y^{2}\right)
$$

with $0<B<1$ fixed, e.g. $B=0.3$. The fixed points (for appropriate $A$ ) are

$$
\begin{aligned}
& x_{1}=y_{1}=\frac{1}{2}(1+B)-\frac{1}{2}\left[(1+B)^{2}+4 A\right]^{1 / 2}, \\
& x_{2}=y_{2}=\frac{1}{2}(1+B)+\frac{1}{2}\left[(1+B)^{2}+4 A\right]^{1 / 2} .
\end{aligned}
$$

The eigenvalues at the fixed point $(x, y)$ are given by $\lambda=y \pm\left[y^{2}-B\right]^{1 / 2}$. Thus the fixed point $\left(x_{1}, y_{1}\right)$ has the following type:

$$
\begin{array}{ll}
A<A_{0} \equiv-\frac{1}{4}(1+B)^{2}, & \text { there are no fixed points, } \\
A=A_{0}, & \text { saddle node, } \lambda=1, B, \\
A_{0}<A \leqq A_{1}, & \text { sink with real positive eigenvalues, } \\
A_{1}<A<A_{2}, & \text { sink with complex eigenvalues, } \\
A_{2}<A<A_{3} \equiv \frac{3}{4}(1+B)^{2}, & \text { sink with real negative eigenvalues, } \\
A=A_{3}, & \text { eigenvalues } \lambda=-1,-\mathrm{B}, \\
A_{3}<A, & \text { hyperbolic with reflection } \lambda_{-}<-1<\lambda_{+}<0 .
\end{array}
$$

As far as the uses for this paper, the important bifurcation occurs at $A=A_{0}$, where a saddle node bifurcation creates a sink for $A$ slightly larger than $A_{0}$. The difference between maps of the real line and the plane is apparent with the eigenvalues becoming complex for $A_{1}<A<A_{2}$.

The next proposition gives a general criterion for the creation of one sink. The basic hypothesis is that there is a box $B$ such that the family $\left\{F_{t}\right\}$ pulls the image of $B$ across $B$ creating a saddle point whose expanding eigenvalue is negative. (There is a reflection in the unstable direction.) See Fig. 5. This result is related to [N5, Lemma 8.2] but the argument there seems to apply only to a generic one parameter family. Also see [N2, Lemma 2]. This proposition is the only modification of the proof in [N4] needed to prove Theorem E. 


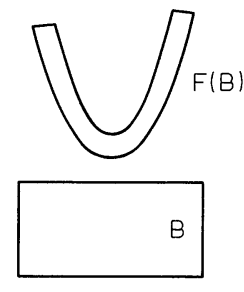

a)

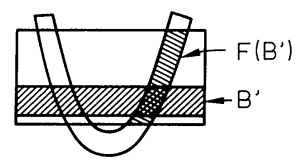

b)

Fig. 5. a The image $F(B)$ for $F=F_{t_{1}}$ lies outside $B$; b The image $F(B)$ for $F=F_{t_{2}}$ crosses $B$ at least twice. The saddle point with reflection is contained in $B^{\prime} \cap F\left(B^{\prime}\right)$

3.3. Proposition. Let $F_{t}(x, y)$ be a one parameter family of $C^{1}$ diffeomorphisms defined in a neighborhood of a box $B=\left\{(x, y):\left|x-x_{0}\right| \leqq \delta_{1}\right.$ and $\left.\left|y-y_{0}\right| \leqq \delta_{2}\right\}$ for $t_{1} \leqq t \leqq t_{2}$. Assume $\left|\operatorname{det} D F_{t}(x, y)\right|<1$ for all $t_{1} \leqq t \leqq t_{2}$ and $(x, y)$ in B. Further assume

(i) $F_{t_{1}} B \cap B=\emptyset$,

(ii) $F_{t_{2}}$ has a fixed point that is a saddle point with reflection, i.e. has real eigenvalues $\lambda \leqq-1$ and $\left|\lambda^{\prime}\right|<1$,

(iii) $F_{t}$ has no fixed points on the boundary of $B$ for all $t_{1} \leqq t \leqq t_{2}$.

Then there is a $t_{0}$ with $t_{1}<t_{0}<t_{2}$ such that $F_{t_{0}}$ has a fixed point sink. If $\operatorname{det} D F_{t}>0$ so $F_{t}$ is orientation preserving, the value $t_{0}$ can be chosen so the eigenvalues have nonzero imaginary part.

3.4. Remark. The way condition (iii) is satisfied in the applications of (3.3) is as follows. Assume $F_{t}(B)$ does not intersect the sides of the box, $\left\{\left(x_{0} \pm \delta_{1}, y\right):\left|y-y_{0}\right|<\delta_{2}\right\}$ for $t_{1} \leqq t \leqq t_{2}$, then there are no fixed points on the sides of $B$. If the top and bottom edges of $B$ always have images outside the box, $F_{t}\left(x, y_{0} \pm \delta_{2}\right)$ is not in $B$ for $t_{1} \leqq t \leqq t_{2}$, then there are no fixed points on the top and bottom of $B$. Thus with these two assumptions, there are no fixed points on the boundary of $B$.

Proof. The proof uses two key ideas: (1) eigenvalues vary continuously and (2) if $F_{t}$ has a fixed point $p(t)$ whose eigenvalues are not equal to +1 then by the implicit function theorem there is an interval $(t-\varepsilon, t+\varepsilon)$ such that for $s$ in this interval $F_{s}$ has a fixed point $p(s)$. Let $p\left(t_{2}\right)$ be the fixed point given by assumption (ii) with eigenvalues $\lambda_{1}\left(t_{2}\right) \leqq-1$ and $\left|\lambda_{2}\left(t_{2}\right)\right|<1$. Let $t_{3}<t \leqq t_{2}$ be the maximal interval of continuing the fixed point $p(t)$ in the interior of $B$. It follows that for $t=t_{3}, F_{t}$ has a fixed point $p\left(t_{3}\right)$ because this is a closed condition. By assumption (iii), $p\left(t_{3}\right)$ can not be on the boundary of $B$, so is in its interior. By assumption (i), for $t=t_{1}, F_{t}$ has no fixed points in $B$, so $t_{1}<t_{3}$. By (2) above it follows that $p\left(t_{3}\right)$ has an eigenvalue equal to +1 .

Either $\lambda_{1}\left(t_{3}\right)=1$ or $\lambda_{2}\left(t_{3}\right)=1$. If $\lambda_{2}\left(t_{3}\right)=1$, then at some intermediate value $t=t_{4}$, $\left|\operatorname{det} D F_{t}(p(t))\right|<\left|\lambda_{2}\left(t_{4}\right)\right|<1$. Because $\left|\operatorname{det} D F_{t}(p(t))\right|=\left|\lambda_{1}\left(t_{4}\right)\right|\left|\lambda_{2}\left(t_{4}\right)\right|$, it follows that also $\left|\lambda_{1}\left(t_{4}\right)\right|<1$ and $p\left(t_{4}\right)$ is a sink. One the other hand if $\lambda_{1}\left(t_{3}\right)=1$, then at some parameter value $t_{5}, \operatorname{Re} \lambda_{1}\left(t_{5}\right)=0$. Since $F_{t}$ is a diffeomorphism, $\lambda_{1}\left(t_{5}\right) \neq 0$ so it must be pure imaginary with $\lambda_{2}\left(t_{5}\right)=-\lambda_{1}\left(t_{5}\right)$. Then for $t=t_{5}, 1>\left|\operatorname{det} D F_{t}(p(t))\right|$ $=\left|\lambda_{2}\left(t_{5}\right)\right|^{2}$ and $p\left(t_{5}\right)$ is a sink.

Note in the case where $\operatorname{det} D F_{t}(p)>0$, the second case must occur so there must be a complex sink. 


\section{Creation of Homoclinic Intersections for Real Analytic Maps}

In this section we reduce the proof of Theorem A to Theorem B, i.e. we show that if $\left\{F_{t}\right\}$ is a real analytic family which creates homoclinic intersections, then it creates odd order homoclinic intersections.

Let $P_{t}$ be the periodic points such that the stable and unstable manifolds of the orbit of $P_{t}$ create homoclinic intersections. There is a $k$ such that for $Q_{t}=F_{t}^{k}\left(P_{t}\right)$, the manifolds $W^{u}\left(Q_{t}, F_{t}\right)$ and $W^{s}\left(P_{t}, F_{t}\right)$ create homoclinic intersections. These manifolds are real analytic for fixed $t$ because $F_{t}$ is real analytic. These manifolds do not coincide for $t_{0}<t \leqq t_{0}+\varepsilon$, because they have two topologically transverse intersections, condition (ii). Because they are real analytic, all the intersections are of finite order. Any intersection of finite order which is topologically transverse is necessarily of odd order. Therefore by condition (ii) there are both positive and negative intersections of odd order.

\section{Infinite Cascade of Sinks: Theorem B}

For simplicity we assume the stable and unstable manifolds which create homoclinic intersections belong to a fixed point $P_{t}$. The case of a periodic point is not much different. See [N4]. We also assume both of the eigenvalues for $P_{t}$ are positive. Again the case where one of these is negative can be handled by replacing $F_{t}$ with $F_{t}^{2}$. Because $0<\operatorname{det} D F_{t_{0}}\left(P_{t_{0}}\right)<1$, the product of the eigenvalues is less than one, $0<\mu \lambda<1$, where $0<\mu<1$ and $1<\lambda$. The tangencies also can occur from various sides and for different branches of $W^{s}\left(P_{t}, F_{t}\right)$ and $W^{u}\left(P_{t}, F_{t}\right)$. Some of these cases imply that $W^{s}\left(P_{t}, F_{t}\right)$ and $W^{u}\left(P_{t}, F_{t}\right)$ already have other intersections for $t=t_{0}$. See the work of Gavrilov and Silnikov, [GS] or [GH]. These differences make no difference in the proof given here. Compare the cases in Figs. 1 and 2 which are different.

For simplicity of discussion we assume that $F_{t}$ can be linearized near $P_{t}$, i.e. there is a neighborhood $U$ of $P_{t}$ and $C^{j}$ coordinates $(x, y)$ on $U$ so that $F_{t}(x, y)$ $=(\mu x, \lambda y)$. Here $j$ is the order of the intersection of stable and unstable manifolds. If $F_{t}$ is $C^{\infty}$ and satisfies nonresonance conditions of the eigenvalues at $P_{t}$, the Sternberg linearization gives such coordinates. In Appendix 5.4 at the end of the section, there is a discussion of how to obtain the necessary estimates without linearizing.

Let $q_{0}$ be the point at which $W^{s}\left(P_{t}, F_{t}\right)$ and $W^{u}\left(P_{t}, F_{t}\right)$ have a tangency at $t=t_{0}$. By looking along the orbit of $q_{0}$, we can assume $q_{0}=\left(x_{0}, 0\right)$ is in $U$ on the local stable manifold of $P_{t_{0}}$. Also by taking $k$ large enough, $q_{1}=F_{t_{0}}^{-k}\left(q_{0}\right)=\left(0, y_{1}\right)$ will also be in $U$.

We next form boxes $B_{n}$ near $q_{0}$ to which Proposition 3.3 can be applied to get a sink. Take $\delta_{1}^{u}, \delta_{1}^{s}, \delta_{0}^{u}, \delta_{0}^{s}>0$ and form

$$
V_{0}=\left\{(x, y):\left|x-x_{0}\right| \leqq \delta_{0}^{s}, 0 \leqq\left(\operatorname{sign} y_{1}\right) y \leqq \delta_{0}^{u}\right\}
$$

and

$$
V_{1}=\left\{(x, y):\left|y-y_{1}\right| \leqq \delta_{1}^{u}, 0 \leqq\left(\operatorname{sign} x_{0}\right) x \leqq \delta_{1}^{s}\right\}
$$




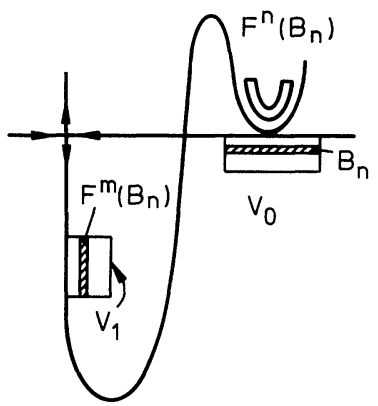

a)

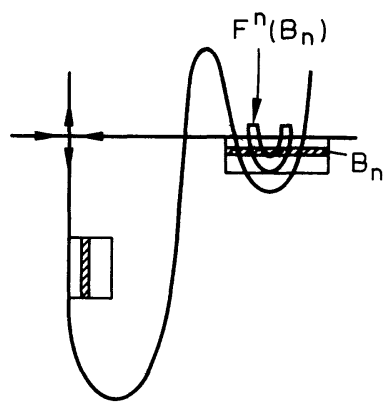

b)

Fig. 6a and b. Location for boxes $B_{n}$ and their images $F_{t}^{m}\left(B_{n}\right)$ and $F_{t}^{n}\left(B_{n}\right)$ for one case of homoclinic tangency. a $t=t_{0} ; \mathbf{b} t>t_{0}$

These choices can be made so that $V_{0} \cap F_{t}\left(V_{0}\right)=\emptyset, V_{1} \cap F_{t}\left(V_{1}\right)=\emptyset, \gamma_{t}^{s} \subset$ boundary $V_{0}$, and $\gamma_{t}^{u} \subset$ boundary $F_{t}^{k}\left(V_{1}\right)$, where $\gamma_{t}^{s} \subset W^{s}\left(P_{t}, F_{t}\right)$ and $\gamma_{t}^{u} \subset W^{u}\left(P_{t}, F_{t}\right)$ are as specified in the condition that $\left\{F_{t}\right\}$ creates odd order homoclinic intersections at $t_{0}$ near $q_{0}$. For $N$ large enough, $n \geqq N$, and $m=n-k$,

$$
B_{n}=\text { component }\left(V_{0} \cap F_{t}^{-m}\left(V_{1}\right)\right)=\left\{(x, y):\left|x-x_{0}\right| \leqq \delta_{1}^{s},\left|y-\lambda^{-m} y_{1}\right|=\lambda^{-m} \delta_{1}^{u}\right\}
$$

is a horizontal strip near $q_{0}$, where we take the component which is the first intersection along $F^{-m}\left(V_{1}\right)$. Then

$$
F^{m}\left(B_{n}\right)=\left\{(x, y):\left|y-y_{1}\right| \leqq \delta_{1}^{u},\left|x-\mu^{m} x_{0}\right| \leqq \mu^{m} \delta_{0}^{s}\right\}
$$

is a vertical strip near $q_{1}$, and $F_{t}^{n}=F_{t}^{k} \circ F_{t}^{m}\left(B_{n}\right)$ is a thin nonlinear box near $q_{0}$ which is parallel to $W^{u}\left(P_{t}, F_{t}\right)$.

The next step is to show Proposition 3.3 implies $F_{t}^{n}$ has a sink in $B_{n}$ for appropriate $t$. Take $t_{0}<T<t_{0}+\varepsilon$. Orienting $W^{s}\left(P_{T}, F_{T}\right)$ and $W^{u}\left(P_{T}, F_{T}\right)$, there is at least one intersection of these manifolds at some $q_{2}$ near $q_{0}$ which is an odd order intersection and the sign of the intersection is different at $q_{2}$ than at $P_{T}$. For large $n, W^{u}\left(P_{T}, F_{T}\right)$ intersects $B_{n}$ monotonically near $q_{2}$ and $F_{T}^{n}\left(B_{n}\right)$ crosses $B_{n}$ monotonically with a reversal in the $y$ direction. We show below in Proposition 5.1 that $F_{t}^{n}$ has a saddle point in $B_{n}$ with eigenvalue $\lambda_{1}<-1$. Remark 3.4 applies to show that there are no fixed points on the boundary of $B_{n}$ as $t$ varies. Also $F_{T}^{n}\left(B_{n}\right) \cap B_{n}=\emptyset$ for either $t=t_{0}$ or $t=t_{0}-\varepsilon$. Therefore Proposition 3.3 applies and there are $t_{n}$ and $\varepsilon_{n}>0$ such that for $t_{n}<t<t_{n}+\varepsilon_{n}, F_{t}$ has a sink $p_{n}$ in $B_{n}$ of period $n$. Note that if $F_{t}^{n}\left(B_{n}\right) \cap B_{n}=\emptyset$ for $t=t_{0}$, then it follows from Proposition 3.3 that $t_{0}<t_{n}$ $<t_{n}+\varepsilon_{n}<T<t_{0}+\varepsilon$. On the other hand if $F_{t}^{n}\left(B_{n}\right) \cap B_{n} \neq \emptyset$ for $t=t_{0}$, then $t_{0}-\varepsilon<t_{n}$ $<t_{n}+\varepsilon_{n}<t_{0}$.

As $n$ increases $B_{n}$ is closer to $W^{s}\left(P_{t}, F_{t}\right)$ and $F_{t}^{n}\left(B_{n}\right)$ is closer to $W^{u}\left(P_{t}, F_{t}\right)$. Therefore for larger $n, T$ can be chosen nearer to $t_{0}$ in the argument above to show there is a sink of period $n$. Therefore as $n$ increases $t_{n}$ approaches $t_{0}$.

All that is left is to show that there is a saddle fixed point of $F_{T}^{n}$ in $B_{n}$ with eigenvalues $\lambda_{1}<-1$ and $\left|\lambda_{2}\right|<1$. This fact follows from the following proposition with $F=F_{T}$ and $P=P_{T}$. 
5.1. Proposition. (a) Assume $F$ is $C^{j}$ and has a fixed point $P$ which has a homoclinic intersection of order $j$ at $q_{2}$ with $j$ odd. Then $q_{2}$ is the limit of hyperbolic saddle point $z_{n}$ of period $n$, where $z_{n}$ is in the box $B_{n}$ as defined above. (b) If the sign of the intersection of $W^{s}(P, F)$ and $W^{u}(P, F)$ is different at $q_{2}$ than at $P$, then the saddle points $z_{n}$ have negative unstable eigenvalue, $\lambda_{1}<-1$.

When the family nondegenerately creates intersections, then $j=1$ and this result follows from the usual transverse homoclinic point result. When $j$ is larger than one, then the image $F^{n}\left(B_{n}\right)$ stretches across $B_{n}$ but the slope is small and so the argument is delicate. In fact if the intersection is $C^{\infty}$ flat, then the result is probably false. Therefore we give the details of this proof which takes most of the rest of the section (up to Remark 5.2).

Proof. We take the case as in part (b) of the proposition, but the other case is similar. Let $q_{2}=\left(x_{2}, 0\right)$ and $q_{3}=F^{-k}\left(q_{2}\right)=\left(0, y_{3}\right)$ be in the linearized neighborhood of $P$. We take $n$ large enough so that $F^{n}\left(B_{n}\right)$ crosses $B_{n}$ monotonically. Therefore there is a horizontal subbox

$$
B_{n}^{\prime}=\left\{(x, y):\left|x-x_{0}\right|<\delta_{0}^{s}, A_{1} \leqq y \leqq A_{2}\right\}
$$

in $B_{n}$ such that (i) $F^{n}\left(B_{n}^{\prime}\right)$ stretches across $B_{n}, F_{2}^{n}\left(x, A_{1}\right) \geqq \lambda^{-m} y_{1}+\lambda^{-m} \delta_{1}^{u} \geqq A_{2}$ and $F_{2}^{n}\left(x, A_{2}\right) \leqq \lambda^{-m} y_{1}-\lambda^{-m} \delta_{1}^{u} \leqq A_{1}$, and (ii) $\partial F_{2}^{n} / \partial y(z)<0$ for $z$ in $B_{n}^{\prime}$. Because of the hyperbolic estimates on $U,\left|\partial F^{n} / \partial x(z)\right|<1$ for $z$ in $B_{n}$.

In this situation it follows that $F^{n}$ has a fixed point $z_{n}=\left(x_{n}, y_{n}\right)$ in $B_{n}^{\prime}$. The existence of $z_{n}$ can be shown using an index argument on $B_{n}^{\prime}$. Alternatively it is possible to show there are (i) a horizontal curve of points in $B_{n}^{\prime}$ which have the $y$ coordinate fixed, and (ii) a vertical curve of points in $B_{n}^{\prime}$ which have the $x$ coordinate fixed. The point $z_{n}$ where these two curves intersect is a fixed point. We need to show that $z_{n}$ is a saddle point with reflection in the unstable direction. Since $|\lambda \mu|<1$, it follows that $\left|\operatorname{det} D F^{n}\left(z_{n}\right)\right|<1$. Thus if we show one of the eigenvalues $\lambda_{1}<-1$, the other eigenvalue $\lambda_{2}$ will have to have $\left|\lambda_{2}\right|<1$.

The graph of $W^{u}(P, F)$ near $q_{2}$ is given by $y=g(x)=a\left(x-x_{2}\right)^{j}+h(x)$. The slope is $g^{\prime}(x)=j a\left(x-x_{2}\right)^{j-1}+h^{\prime}(x)$. As a function of $y$, the slope at $z_{n}=\left(x_{n}, y_{n}\right)$ is about $j a\left(y_{n} / a\right)^{(j-1) / j}=j a^{1 / j} y_{n}^{1-1 / j} \equiv \xi_{n}$. Let $\xi_{n}^{+}=\left|\xi_{n}\right|$. We need an estimate below on $\xi_{n}^{+}$. Since $\left(\left|y_{1}+\delta_{1}^{u}\right|\right) \lambda^{-m} \geqq\left|y_{n}\right| \geqq\left|y_{1}-\delta_{1}^{u}\right| \lambda^{-m}$, there is a $C$ independent of $n$ such that $2 C \lambda^{-m+m / j} \geqq \xi_{n}^{+} \geqq C \lambda^{-m+m / j}$. To find the unstable eigenvector we take the sector of vectors whose slopes are about $\xi_{n}$,

$$
S\left(z_{n}\right)=\left\{v=\left(v_{1}, v_{2}\right): \eta^{-1}<v_{2} /\left(v_{1} \xi_{n}\right)<\eta\right\},
$$

where $\eta>1$ is independent of $n$. Thus for $v$ in $S\left(z_{n}\right)$ the slope of $v$ is between $\eta^{-1} \xi_{n}$ and $\eta \xi_{n}$. We show that for $v$ in $S\left(z_{n}\right), v^{\prime \prime}=D F^{n}\left(z_{n}\right) v$ is also in $S\left(z_{n}\right)$ and $\left\|v^{\prime \prime}\right\|>\|v\|$. Thus there is a vector $v_{0}$ in $S\left(z_{n}\right)$ such that $D F^{n}\left(z_{n}\right) v_{0}=\lambda_{1} v_{0}$ and $\left|\lambda_{1}\right|>1$. Since $D F^{n}\left(z_{n}\right)$ reverses the second coordinate, $\lambda_{1}<-1$.

The proof of these facts about vectors $v$ in $S\left(z_{n}\right)$ is like the proofs of Lemmas 7.5 and 7.6 below. There we prove there is a hyperbolic invariant set under slightly different assumptions. Let $v=\left(v_{1}, v_{2}\right)$ be in $S\left(z_{n}\right), v^{\prime}=\left(v_{1}^{\prime}, v_{2}^{\prime}\right)=D F^{m}\left(z_{n}\right) v$ $=\left(\mu^{m} v_{1}, \lambda^{m} v_{2}\right)$, and $v^{\prime \prime}=\left(v_{1}^{\prime \prime}, v_{2}^{\prime \prime}\right)=D F^{n}\left(z_{n}\right) v$. The linear estimates in $U$ and the bound 


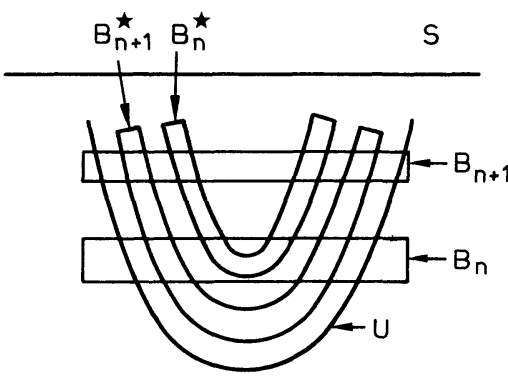

a)
S

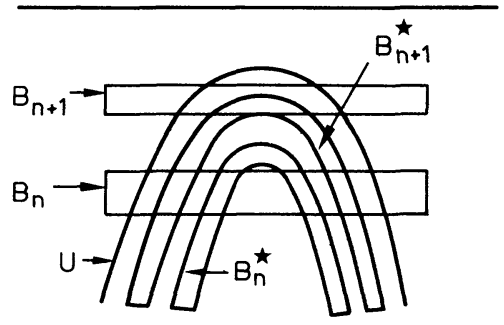

b)

Fig. 7a and $\mathbf{b}$. The stable and unstable manifolds of $P_{t}$ are labelled $S$ and $U$, respectively. The images $F_{t}^{J}\left(B_{j}\right)$ are labelled $B_{j}^{*}$. a $F_{t}$ is as in Fig. 5 with $t_{n}<t<t_{n}+\varepsilon_{n}$ : b $F_{t}$ is as in Fig. 2 with $t_{n+1}<t$ $<t_{n+1}+\varepsilon_{n+1}$

on the slope of $v$ show that $v^{\prime}$ is nearly vertical for $n$ large:

$$
\begin{aligned}
\left|v_{2}^{\prime} / v_{1}^{\prime}\right| & =\lambda^{m} \mu^{-m}\left|v_{1} / v_{2}\right| \geqq \lambda^{m} \mu^{-m} \eta^{-1} \xi_{n}^{+} \\
& \geqq \lambda^{n} \mu^{-m} \eta^{-1} C \lambda^{-m+m / j} \\
& \geqq \eta^{-1} C \mu^{-m} \lambda^{m / j} .
\end{aligned}
$$

Then $v^{\prime \prime}$ has slope about $\xi_{n}$, in fact the slope is between $\eta^{-1} \xi_{n}$ and $\eta \xi_{n}$ for large $n$. Thus $v^{\prime \prime}$ lies in $S\left(z_{n}\right)$. Moreover using the fact there is a constant $C_{1}>0$ such that for any $z$ near $q_{3}$ and for any $v^{\prime},\left\|v^{\prime \prime}\right\|=\left\|D F^{k}(z) v^{\prime}\right\| \geqq C_{1}\left\|v^{\prime}\right\|$; it follows that $\left\|v^{\prime \prime}\right\|>\|v\|$ :

$$
\begin{aligned}
\left\|v^{\prime \prime}\right\| & \geqq C_{1}\left\|v^{\prime}\right\| \geqq C_{1}\left|v_{2}^{\prime}\right| \geqq C_{1} \lambda^{m}\left|v_{2}\right| \geqq C_{1} \lambda^{m} \eta^{-1} \xi_{n}\left(1+\eta^{-2} \xi_{n}^{2}\right)^{-1 / 2}\|v\| \\
& \geqq C_{1} \lambda^{m} \eta^{-1} \xi_{n}\|v\| / 2 \geqq C_{1} \lambda^{m} \eta^{-1} C \lambda^{-m+m / j}\|v\| / 2 \geqq \eta^{-1} C_{1} C \lambda^{m / j}\|v\| / 2 \geqq\|v\|
\end{aligned}
$$

for large enough $m$. This completes the proof of Proposition 5.1 and Theorem D.

5.2. Remark. The intervals of parameter values $t_{n}<t<t_{n}+\varepsilon_{n}$ obtained above for the sinks $p_{n}$ of period $n$ do not overlap for large $n, n \geqq N$, at least in the case where $\left\{F_{t}\right\}$ nondegenerately creates homoclinic intersections. In the case of Figs. 7a and 6 , by the time $t>t_{n}$, where the sink $p_{n}$ exists and $F_{t}^{n}\left(B_{n}\right) \cap B_{n} \neq \emptyset$, the geometry forces $F_{t}^{n+1}\left(B_{n+1}\right) \cap B_{n+1}$ to be a complete horseshoe and the periodic point $p_{n+1}$ has become a saddle point with reflection. Therefore $t_{0}<\ldots<t_{n+1}<t_{n+1}+\varepsilon_{n+1}$ $<t_{n}<t_{n}+\varepsilon_{n}$ for $n \geqq N$. In other cases such as Figs. $7 \mathrm{~b}$ and 2 the geometry does not force the situation but a distance estimate does. The boxes $B_{n}$ and $B_{n+1}$ are roughly $\lambda^{-m}\left(1-\lambda^{-1}\right)$ distance apart where $m=n-k$, while the images $F_{t}^{n}\left(B_{n}\right)$ and $F_{t}^{n+1}\left(B_{n+1}\right)$ are roughly $\mu^{m}(1-\mu)$ distance apart. Since $\mu<\lambda^{-1}$, by the time $t_{n+1}<t$ $<t_{n+1}+\varepsilon_{n+1}$, where the sink $p_{n+1}$ exists and $F_{t}^{n+1}\left(B_{n+1}\right) \cap B_{n+1} \neq \emptyset, F_{t}^{n}\left(B_{n}\right) \cap B_{n}$ is a complete horseshoe and $p_{n}$ is a saddle point with reflection. Therefore $t_{0}>\ldots>t_{n+1}>t_{n+1}+\varepsilon_{n+1}>t_{n}>t_{n}+\varepsilon_{n}$ for $n \geqq N$. Therefore in any case the above argument can prove the existence of an infinite cascade of sinks, but it is not sufficient to prove there is one parameter value with an infinite number of sinks. The extra needed ingredient in the proof is the thickness of the Cantor set of stable manifolds of a basic set. The next section defines the thickness of a Cantor set and then Sect. 7 applies it to the stable manifolds. 
5.3. Remark. The result of Curry and Johnson mentioned in Remark 2.3, [CJ], follows from the distance of the boxes $B_{n}$ from $W^{s}\left(P_{t}, F_{t}\right)$ and $F_{t}^{n}\left(B_{n}\right)$ from $W^{u}\left(P_{t}, F_{t}\right)$. There distances are roughly $C_{1} \lambda^{-m}$ and $C_{2} \mu^{m}$ for $m=n-k$. If these boxes are pulled across at a linear rate then $t_{n}-t_{0} \simeq C_{3}\left(C_{1} \lambda^{-m} \pm C_{2} \mu^{m}\right) \simeq C \lambda^{-m}$ since $\lambda^{-1}>\mu$. Thus

$$
\lim _{n \rightarrow \infty}\left(t_{n}-t_{n-1}\right) /\left(t_{n+1}-t_{n}\right)=\lambda .
$$

See $[\mathrm{CJ}]$. Also compare with the result in [GS2] about the parameter value having hyperbolic sets.

5.4. Appendix. Estimates without linearizing. Because we use the Taylor expansion of the graph of $W^{u}\left(P_{T}, F_{T}\right)$, we need $C^{j}$ coordinates. Unless we make nonresonance assumptions on the eigenvalues, it is not possible to linearize the system. However it is still possible to find coordinates on which there are hyperbolic estimates which are good enough to prove $z_{n}$ is a saddle point with reflection in the unstable direction.

There is a neighborhood $u$ of $P=P_{T}$ and $C^{j}$ coordinates $(x, y)$ on $U$ so that the local stable and unstable manifolds of $P$ are given by $\operatorname{comp}\left(W^{s}(P, F) \cap U\right)$ $=\left\{(x, 0) \cap U\right.$ and $\operatorname{comp}\left(W^{u}(P, F) \cap U\right)=\{(0, y)\} \cap U$. Also

$$
D F(x, y)=\left(\begin{array}{ll}
b_{11} & b_{12} \\
b_{21} & b_{22}
\end{array}\right),
$$

where $\mu-\varepsilon \leqq b_{11} \leqq \mu+\varepsilon, \lambda-\varepsilon \leqq b_{22} \leqq \lambda+\varepsilon,\left|b_{12}\right| \leqq \varepsilon|x|$, and $\left|b_{21}\right| \leqq \varepsilon|y|$. These last two estimates follow because $b_{12}(0, y)=0,\left(W^{u}=\{(0, y)\}\right)$, so

$$
\left|b_{12}(x, y)\right| \leqq|x| \sup \left|\partial^{2} F_{1} / \partial x \partial y\right|
$$

by the Mean Value Theorem. By a change of scale the second partial derivatives can all be made less than $\varepsilon$. The estimate on $b_{21}(x, y)$ is similar.

We need to show that $v^{\prime}=\left(v_{1}^{\prime}, v_{2}^{\prime}\right)=D F^{m}\left(z_{n}\right) v$ is nearly vertical. The fact that $\left\|v^{\prime \prime}\right\|=\left\|D F^{n}\left(z_{n}\right) v\right\|>\|v\|$ follows directly as before. If $\left(x_{r}, y_{r}\right)=F^{r}\left(z_{n}\right)$ for $0 \leqq r \leqq m$, then

$$
\left|x_{r}\right|=\left|F_{1}\left(x_{r-1}, y_{r-1}\right)-F_{1}\left(0, y_{r-1}\right)\right|<\left|x_{r-1}\right| \sup \left|\partial F_{1} / \partial x\right| \leqq(\mu+\varepsilon)\left|x_{r-1}\right| \leqq(\mu+\varepsilon)^{r}\left|x_{0}\right|
$$

by induction. Similarly $\left|y_{r}\right|<\left|y_{m}\right|(\lambda-\varepsilon)^{r-m}$. Let $D F\left(x_{r}, y_{r}\right)=\left(b_{i j}^{r}\right)$. Then

$$
\begin{gathered}
\mu-\varepsilon \leqq b_{11}^{r} \leqq \mu+\varepsilon, \quad\left|b_{12}^{r}\right| \leqq \varepsilon\left|x_{0}\right|(\mu+\varepsilon)^{r}, \\
\left|b_{21}^{r}\right| \leqq \varepsilon\left|y_{m}\right|(\lambda-\varepsilon)^{r-m}, \quad \lambda-\varepsilon \leqq b_{22}^{r} \leqq \lambda+\varepsilon .
\end{gathered}
$$

It suffices to consider $v$ in $S\left(z_{n}\right)$ with $v_{1}=1$. Let $v^{k}=\left(v_{1}^{r}, v_{2}^{r}\right)=D F^{r}\left(z_{n}\right) v$. We know that $\eta^{-1} \xi_{n}^{+} \leqq\left|v_{2}\right| \leqq \eta \varepsilon_{n}^{+}$and $\xi_{n}^{+}=A \lambda^{-m+m / j}$ with $C \leqq A \leqq 2 C$. We prove by induction on $r$ that

$$
\begin{gathered}
\eta^{-1} A(\lambda-2 \varepsilon)^{r-m+m / j} \leqq\left|v_{2}^{r}\right| \leqq A(\lambda+2 \varepsilon)^{r-m+m / j}, \\
\left|v_{1}^{r}\right| \leqq[(\mu+2 \varepsilon)(\lambda+2 \varepsilon)]^{r}
\end{gathered}
$$

Once we prove these inequalities then

$$
\left|v_{2}^{\prime}\right| /\left|v_{1}^{\prime}\right|=\left|v_{2}^{m}\right| /\left|v_{1}^{m}\right| \geqq \eta^{-1} A(\lambda-2 \varepsilon)^{m / j}[(\mu+2 \varepsilon)(\lambda+2 \varepsilon)]^{-m}
$$

is arbitrarily large as $m$ goes to infinity (for small enough $\varepsilon$ ). 
To show (5.5) for $v_{1}^{r}$

$$
\begin{aligned}
\left|v_{1}^{r+1}\right| & \leqq\left|b_{11}^{r}\right|\left|v_{1}^{r}\right|+\left|b_{12}^{r}\right|\left|v_{2}^{r}\right| \\
& \leqq(\mu+\varepsilon)[(\mu+2 \varepsilon)(\lambda+2 \varepsilon)]^{r}+\varepsilon\left|x_{0}\right|(\mu+\varepsilon)^{r} A(\lambda+2 \varepsilon)^{r-m+m / j} \\
& \leqq[(\mu+2 \varepsilon)(\lambda+2 \varepsilon)]^{r+1}\left\{(\lambda+2 \varepsilon)^{-1}+\varepsilon\left|x_{0}\right| \eta A(\mu+2 \varepsilon)^{-1}(\lambda+2 \varepsilon)^{-1-m+m / j}\right\} \\
& \leqq[(\mu+2 \varepsilon)(\lambda+2 \varepsilon)]^{r}
\end{aligned}
$$

for $\varepsilon$ small enough. Similarly for $v_{2}^{r}$,

$$
\begin{aligned}
\left|v_{2}^{r+1}\right| & \geqq-\left|b_{21}\right|\left|v_{1}^{r}\right|+\left|b_{22}\right|\left|v_{2}^{r}\right| \\
& \geqq-\varepsilon\left|y_{m}\right|(\lambda-\varepsilon)^{r-m}[(\mu+2 \varepsilon)(\lambda+2 \varepsilon)]^{r}+(\lambda-\varepsilon) \eta^{-1} A(\lambda-2 \varepsilon)^{r-m+m / j} \\
& \geqq \eta^{-1} A(\lambda-2 \varepsilon)^{r+1-m+m / j}\left\{-\varepsilon\left|y_{m}\right|(\lambda-2 \varepsilon)^{-1-m / j}+(\lambda-\varepsilon)(\lambda-2 \varepsilon)^{-1}\right\} \\
& \geqq \eta^{-1} A(\lambda-2 \varepsilon)^{r+1-m+m / j}
\end{aligned}
$$

for $\varepsilon$ small enough. The upper estimate for $\left|v_{2}^{r+1}\right|$ is similar. This completes the necessary modifications without linearization.

\section{Persistent Intersections of Cantor Sets}

Given two Cantor sets $A^{1}$ and $A^{2}$ in the line $\mathbb{R}$, we want a criterion to imply that $A^{1} \cap A^{2} \neq \emptyset$. The relevant condition is the thickness as defined by Newhouse in [N1]. It is related to the Hausdorff dimension of the set, but different. See [N4, p. 107].

A Cantor set $A$ in the line can be represented as $A=\bigcap_{i \geqq 0} A_{i}$, where $A_{0}$ is the smallest interval containing $A$ and $A_{i}=A_{0}-U_{1 \leqq j \leqq i} U_{j}$ and $U_{j}$ are open intervals. Such a sequence of sets $\left\{A_{i}\right\}$ is called a defining sequence of $A$. It is obtained by specifying an ordering of the gaps removed from $A_{0}$ to form $A$.

The thickness is the ratio of the length of intervals left in $A_{i}$ to the length of the adjacent gap $U_{i}$. Let $I_{i j}$ for $j=1,2$ be the two components of $A_{i}$ on either side of the gap $U_{i}$. Let $\ell J$ be the length of an interval $J$. The thickness of a defining sequence is defined by

$$
\tau\left(\left\{A_{i}\right\}\right)=\inf \left\{\ell\left(I_{i j}\right) / \ell\left(U_{i}\right): i \geqq 1, j=1,2\right\} .
$$

This thickness depends on the choice of the defining sequence. The thickness of the Cantor set is defined as the thickness for the best choice of a defining sequence:

$$
\tau(A)=\sup \left\{\tau\left(\left\{A_{i}\right\}\right):\left\{A_{i}\right\} \text { is a defining sequence for } A\right\} .
$$

For a Cantor set $A_{\alpha}$ formed by removing the middle $\alpha$ of the remaining intervals at each step, the intervals at the $k^{\text {th }}$ step have length $((1-\alpha) / 2)^{k}$. The gaps formed at the $k^{\text {th }}$ step are each $\alpha$ of the length of the intervals at the $(k-1)^{\text {th }}$ step, or $\alpha((1-\alpha) / 2)^{k-1}$. Therefore the ratio is always

$$
\frac{\left(\frac{1-\alpha}{2}\right)^{k}}{\alpha\left(\frac{1-\alpha}{2}\right)^{k-1}}=\frac{1-\alpha}{2 \alpha} \text {. }
$$




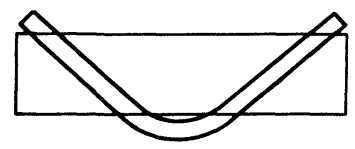

a)

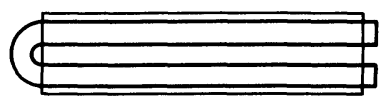

b)

Fig. 8. a $F_{t}^{n}\left(B_{n}\right)$ is shown crossing $B_{n}$ for $t$ as in Proposition 7.1: b $F_{l}^{-n}\left(B_{n}\right)$ is shown crossing $B_{n}$

This defining sequence is actually the best choice so $\tau\left(A_{\alpha}\right)=(1-\alpha) / 2 \alpha$. In particular $\tau\left(A_{1 / 3}\right)=1$ for the middle third Cantor set. Also note $0<\tau(A)<\infty$ and $\tau\left(A_{\alpha}\right)$ goes to infinity as $\alpha$ goes to zero.

The reason for the above definition of thickness is the following lemma which is proven in [N4, Lemma 4, p. 107].

6.1. Lemma. Let $A^{1}$ and $A^{2}$ be two Cantor sets in $\mathbb{R}$ with $A^{1}$ not contained in any gap of $A^{2}$ and vice versa. In particular if $A_{0}^{j}$ is the smallest interval containing $A^{j}$, then assume int $A_{0}^{1} \cap \operatorname{int} A_{0}^{2} \neq \emptyset$. Further assume $\tau\left(A^{1}\right) \tau\left(A^{2}\right)>1$. Then $A^{1} \cap A^{2} \neq \emptyset$. In fact if $\left\{A_{i}^{j}\right\}$ are defining sequences for $A^{j}$ for $j=1,2$ such that $\tau\left(\left\{A_{i}^{1}\right\}\right) \tau\left(\left\{A_{i}^{2}\right\}\right)>1$, then for each $i \geqq 1, \operatorname{int}\left(A_{i}^{1} \cap A_{i}^{2}\right) \neq \emptyset$.

\section{Thick Cantor Sets of Stable Manifolds from a Nondegenerate Tangency}

If $\Lambda$ is a hyperbolic set for $F$, the stable thickness of $\Lambda, \tau^{s}(\Lambda)$, is defined by $\tau^{s}(\Lambda)$ $=\limsup _{\varepsilon \rightarrow 0}\left\{\tau\left(\gamma_{\varepsilon} \cap W^{\mathrm{s}}(\Lambda)\right)\right\}$, where $\gamma$ is any $C^{1}$ arc transverse to the stable manifolds $W^{s}(\Lambda)$ at $q$ and $\gamma_{\varepsilon}$ is the arc of length $\varepsilon$ in $\gamma$ centered at $q$. The result [N4, Proposition 5] shows this definition of thickness is independent of $\gamma$ and $q$ for $F$ which is $C^{2}$ and $\gamma$ is $C^{1}$. Thus it is well defined. The unstable thickness, $\tau^{u}(\Lambda)$, is defined in a similar manner.

It is not hard to construct a diffeomorphism which has a horseshoe $\Lambda$ with $W^{s}(\Lambda) \cap \gamma^{s}$ and $W^{u}(\Lambda) \cap \gamma^{u}$ any desired middle $\alpha$ and $\beta$ Cantor sets. Thus $\tau^{s}(\Lambda)$ and $\tau^{u}(\Lambda)$ are arbitrarily large. Moreover it can be done so that the fact $\tau^{s}(\Lambda) \tau^{u}(\Lambda)>1$ implies there is a persistent tangency of $W^{\mathrm{s}}(\Lambda)$ and $W^{u}(\Lambda)$. See [N1] and [N5, pp. 103-104].

In this section and the next, we show that whenever a family nondegenerately creates a homoclinic intersections, then there are created hyperbolic invariant sets $\Lambda(t)$ which have $\tau^{s}(\Lambda(t))$ arbitrarily large and a persistent tangency. The idea of the construction is as follows. In this section the parameter value $t_{n}^{*}$ is chosen carefully so that there is a hyperbolic invariant set $\Lambda_{n}\left(t_{n}^{*}\right)$ in $B_{n}$ with $\tau^{s}\left(\Lambda_{n}\left(t_{n}^{*}\right)\right)$ arbitrarily large. The parameter value must be chosen so $F_{t}^{n}\left(B_{n}\right)$ comes out the bottom of the box $B_{n}$ enough so the maximal invariant set in $B_{n}$ has a hyperbolic structure, $A_{n}(t)$ $=\bigcap_{n} F_{t}^{n}\left(B_{n}\right)$. On the other hand $t$ must be chosen so that most of $F_{t}^{n}\left(B_{n}\right)$ lies in $B_{n}$ so $\Lambda_{n}\left(t_{n}^{*}\right)$ has large stable thickness. In terms of the quantities in Fig. 9 below, the gap $g$ must be large enough to make $\Lambda_{n}\left(t_{n}^{*}\right)$ hyperbolic while the gap $g^{\prime}$ must be small enough to make $\tau^{s}\left(\Lambda_{n}\left(t_{n}^{*}\right)\right)$ large. However for $t=t_{n}^{*}$ there might not be any homoclinic tangencies because the local extreme points of $W^{u}\left(\Lambda_{n}\left(t_{n}^{*}\right)\right)$ relative to $W^{s}\left(\Lambda_{n}\left(t_{n}^{*}\right)\right)$ lie away from $W^{s}\left(\Lambda_{n}\left(t_{n}^{*}\right)\right)$. In the next section, $t_{n}^{*}$ is decreased to $t_{n}^{* *}$ to 


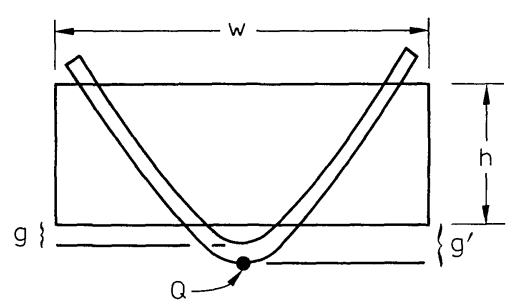

Fig. 9. The gaps $g$ and $g^{\prime}$ are indicated as well as the height $h$ and width $w$. The point $Q$ is $\left(x_{n}(t), y_{n}(t)\right)$

cause the extreme points of $W^{u}\left(\Lambda_{n}\left(t_{n}^{*}\right)\right)$ to intersect the $W^{s}\left(\Lambda_{n}\left(t_{n}^{*}\right)\right)$ and so to cause a persistent tangency. The value $t=t_{n}^{* *}$ is chosen carefully so that $F_{t}^{n}\left(B_{n}\right)$ does not come out the bottom of $B_{n}$ but there is still a smaller hyperbolic set $A_{n}^{\prime}(t)$ in $B_{n}$ with $\tau^{s}\left(\Lambda_{n}^{\prime}(t)\right)$ large. There is a $C^{1}$ curve $\gamma$ along which $W^{u}\left(\Lambda_{n}^{\prime}(t)\right)$ has extrema relative to $W^{s}\left(\Lambda_{n}^{\prime}(t)\right)$. The value $t=t_{n}^{* *}$ is chosen so that $\gamma \cap W^{u}\left(\Lambda_{n}^{\prime}(t)\right)$ and $\gamma \cap W^{s}\left(\Lambda_{n}^{\prime}(t)\right)$ overlap along $\gamma$ and the product thickness is larger than one. Thus there is a tangency of $W^{u}\left(\Lambda_{n}^{\prime}(t)\right)$ and $W^{s}\left(\Lambda_{n}^{\prime}(t)\right)$ at some point of $\gamma$. This tangency persists for $t$ near $t_{n}^{* *}$. More details of the construction will follow.

It is easier to use coordinates in which $F_{t}$ is linear. We need to have $C^{2}$ coordinates because we look at the curvature of $W^{u}\left(P_{t}, F_{t}\right)$ near $q_{0}$. If $F_{t}$ is $C^{\infty}$ and the eigenvalues at $P_{t}$ satisfy nonresonance conditions, then Sternberg linearization gives $C^{2}$ coordinates in which $F_{t}$ is linear near $P_{t}$. Even without the nonresonance conditions, Newhouse showed that if $F_{t}$ is $C^{3}$ it is possible to find coordinates on $U$ that are $C^{1}$ everywhere, are $C^{2}$ off $W^{u}\left(P_{t}, F_{t}\right)=\{(0, y)\}$, and such that in these coordinates $F_{t}$ is linear, $F_{t}(x, y)=(\mu x, \lambda y)$. See [N4, pp. 124-126]. The proof uses the fact that $\mu \lambda<1$. The main idea is that with these assumptions there are $C^{2}$ line bundles in the stable and unstable directions off $W^{u}\left(P_{t}, F_{t}\right)$. We use these coordinates in this and the next section.

7.1. Proposition. [N4, Lemma 7.] For suitably chosen $t>t_{n}+\varepsilon_{n}$, the hyperbolic basic set $\Lambda_{n}(t)$ created in $F_{t}^{n}\left(B_{n}\right) \cap B_{n}$ has arbitrarily large $\tau^{s}\left(\Lambda_{n}(t)\right)$.

Proof. The idea of the proof is that because $\mu \lambda<1, F_{t}^{n}\left(B_{n}\right)$ is much thinner than it is long. Therefore by carefully choosing the dimensions of $B_{n}$, for value $t$ for which $F_{t}^{n}\left(B_{n}\right) \cap B_{n}$ first has two components, the length of the bend $F_{t}^{n}\left(B_{n}\right)-B_{n}$ is much smaller than the length of the components $F_{t}^{n}\left(B_{n}\right) \cap B_{n}$. This ratio is roughly the same as the ratio of the height of the component of $F_{t}^{n}\left(B_{n}\right) \cap B_{n}$ to the height of the middle gap in $B_{n}-F_{t}^{-n}\left(B_{n}\right)$, which in turn can be shown to be roughly the stable thickness of the hyperbolic set for $F_{t}^{n}$ in $B_{n}, A_{n}(t)=\bigcap_{j=-\infty}^{\infty} F_{t}^{j n}\left(B_{n}\right)$. See [N4, pp. $121-138]$ for the proof. Since this ratio is arbitrarily large for large $n, \tau^{s}\left(\Lambda_{n}(t)\right)$ is arbitrarily large for suitably chosen $t$ and large $n$. Also $\Lambda_{n}(t)$ has a dense orbit because it is conjugate to a shift on two symbols. Therefore $\Lambda_{n}(t)$ is a basic set.

Let $g$ be the gap by which $F_{t}^{n}\left(B_{n}\right)$ clears the bottom of $B_{n}$ at the bottom bend

$$
\begin{aligned}
g= & \inf \left\{\sup \left\{\text { distance }\left(F_{t}^{n}\left(x_{0}, y\right), B_{n}\right):\left(x_{0}, y\right) \in B_{n} x_{0} \text { fixed }\right\}: x_{0}\right. \text { varies } \\
& \text { with } \left.\left(x_{0}, y^{\prime}\right) \in B_{n}\right\} .
\end{aligned}
$$


See Fig. 9. Let $g^{\prime}$ be the distance the lowest point on the bend is from the bottom of $B_{n}, g^{\prime}=\sup \left\{\sup \left\{\right.\right.$ distance $\left(F_{t}^{n}\left(x_{0}, y\right), B_{n}\right):\left(x_{0}, y\right) \in B_{n} x_{0}$ fixed $\}: x_{0}$ varies with $\left.\left(x_{0}, y^{\prime}\right) \in B_{n}\right\}$.

The dimensions of the box $B_{n}$ and bounds on $g$ and $g^{\prime}$ (hence choice of $t$ ) have to be chosen to satisfy four conditions :

(7.2a) the height $h$ of $B_{n}$ must be big enough for $F_{t}^{n}\left(B_{n}\right)$ to stretch across $B_{n}$ from top to bottom and back to the top again,

(7.2b) the width $w$ must be large enough so that $F_{t}^{n}\left(B_{n}\right)$ comes out the top of $B_{n}$ and not the sides,

(7.2c) the bound on $g^{\prime}$ must be small enough to insure that for $x^{\prime}$ fixed the length of a component of $F_{t}^{n}\left\{\left(x^{\prime}, y\right):\left(x^{\prime}, y\right) \in B_{n}\right\} \cap B_{n}$ must be long relative to the components of $F_{t}^{n}\left\{\left(x^{\prime}, y\right) \in B_{n}\right\}-B_{n}$ so that $\tau^{s}\left(\Lambda_{n}(t)\right)$ is arbitrarily large,

(7.2d) the gap $g$ must be large enough to make the slope of $\partial F_{t}^{n} / \partial y(q)$ large enough for $q$ in $B_{n} \cap F_{t}^{n}\left(B_{n}\right)$ to prove the set $\Lambda_{n}(t)$ has a hyperbolic structure.

Let $m=n-k$ and $H_{t}=F_{t}^{k}$ be the map from $F_{t}^{m}\left(B_{n}\right) V_{1}$ to $V_{0}$. Thus $F_{t}^{n}$ from $B_{n}$ to $V_{0}$ is given by $F_{t}^{n}(x, y)=H_{t}\left(\lambda^{m} x, \mu^{m} y\right)$. For $t=t_{0}, H_{t}\left(0, y_{1}\right)=\left(x_{0}, 0\right)$ corresponds to the nondegenerate tangency. Let

$$
\begin{aligned}
d_{1} & =\left|\partial H_{t} / \partial y\left(0, y_{1}\right)\right|=\left|\partial H_{1 t} / \partial y\left(0, y_{1}\right)\right|, \\
d_{2} & =\left|\partial H_{t} / \partial x\left(0, y_{1}\right)\right| \quad \text { and } \\
y & =a\left(x-x_{n}(t)\right)^{2}+y_{n}(t)
\end{aligned}
$$

be such that for $x^{\prime}$ fixed $\left\{H\left(x^{\prime}, y\right):\left(x^{\prime}, y\right) \in F_{t}^{m}\left(B_{n}\right)\right\}$ is $C^{2}$ near $\left\{(x, y): y=a\left(x-x_{n}(t)\right)^{2}\right.$ $\left.+y_{n}(t)\right\}$. Here $\left(x_{n}(t), y_{n}(t)\right)$ is the point where $y$ has the smallest value on $F_{t}^{n}\left(B_{n}\right)$. Letting $\alpha$ be an arbitrarily small positive constant which is different for different quantities, the choices which work to satisfy (7.2) are

$$
\begin{array}{ll}
w=\frac{(4+\alpha)}{a d_{1}} \lambda^{-m}, & h=\frac{w \lambda^{-m}}{d_{1}-\alpha}=\frac{(4+\alpha)}{a d_{1}^{2}} \lambda^{-2 m}, \\
g \geqq a^{-1}\left(\mu \lambda^{-1}\right)^{2 m-2 \alpha m}, & g^{\prime} \leqq w 10 d_{2} \mu^{m}=h 10 d_{2}\left(d_{1}-\alpha\right)(\mu \lambda)^{m} .
\end{array}
$$

The choices for $w$ and $h$ are the same as [N4, p. $126(1)-(4)]$ but the bounds for $g$ and $g^{\prime}$ are different and more specific. In [N4], $g$ and $g^{\prime}$ are assumed to be $\alpha w \lambda^{-m}$, where $\alpha$ is a small unspecified constant. Our lower bound on $g$ makes it clear how large it must be in order to show that $\Lambda_{n}(t)$ has a hyperbolic structure. This bound is important in the next section to know, for a nearby value of $t$, how much of $B_{n}$ must be removed to get a hyperbolic set $\Lambda_{n}^{\prime}(t)$. As a final remark, the only place the lower bound of $g$ is used is in the proof of Lemma 7.5 which shows there are invariant sectors in the unstable direction. The rest of this section sketches the proof that the choices (7.4) imply (7.2).

Proof of 7.2a. For $x^{\prime}$ fixed, on either side of the minimum the curve $F_{t}^{n}\left\{\left(x^{\prime}, y \in B_{n}\right)\right\}$ is monotone so its length is less than the sum of the change in the $x$ value plus the change in the $y$ value. Since $F_{t}^{n}(x, y)=H_{t}\left(\mu^{m} x, \lambda^{m} y\right)$, to show $F_{t}^{n}\left(B_{n}\right)$ can stretch across $B_{n}$ it suffices for

$$
\left(d_{1}-\alpha\right) \lambda^{m} h \geqq 2(w / 2)+2\left(h+g^{\prime}\right)
$$


or

$$
h \geqq\left[\left(d_{1}-\alpha\right) \lambda^{m}-2\right]^{-1} w\left(1+10 d_{2} \mu^{m}\right) .
$$

Thus the value $h=w\left(d_{1}-\alpha\right)^{-1} \lambda^{-m}$ in (6.4) suffices when the $\alpha$ for $\lambda$ is different and slightly larger than the $\alpha$ for $g^{\prime}$.

Proof of 7.2b. To show $F_{t}^{n}\left(B_{n}\right)$ comes out the top of $B_{n}$ rather than the sides, it suffices to show that $a(w / 2)^{2}$ is larger than $h+g^{\prime}=w \lambda^{-m}\left[\left(d_{1}-\alpha\right)^{-1}+10 d_{2}(\mu \lambda)^{m}\right]$. Thus it suffices for

$$
w \geqq \lambda^{-m} 4 a^{-1}\left[\left(d_{1}-\alpha\right)^{-1}+10 d_{2}(\mu \lambda)^{m}\right] .
$$

Thus $w=(4+\alpha) \lambda^{-m} a^{-1} d_{1}^{-1}$ as in (7.4) is adequate to satisfy (7.2b).

Proof of $7 c$. We need to show for $x^{\prime}$ fixed the length of a component of $F_{t}^{n}\left\{\left(x^{\prime}, y\right) \in B_{n}\right\} \cap B_{n}$ is long compared with the length of the gap $F_{t}^{n}\left\{\left(x^{\prime} y\right) \in B_{n}\right\}-B_{n}$. The length of the gap is less than the sum of the change in its $x$ coordinate plus the change in its $y$ coordinate. Thus it is less than

$$
g^{\prime}+\left(g^{\prime} / a\right)^{1 / 2} \leqq w 10 d_{2} \mu^{m}+\left(w 10 d_{2} \mu^{m} a^{-1}\right)^{1 / 2} .
$$

The length of a component of $F_{t}^{n}\left\{\left(x^{\prime}, y\right) \in B_{n}\right\} \cap B_{n}$ is greater than the change in its $x$-coordinate. Thus it is greater than

$$
(h / a)^{1 / 2}-\left(g^{\prime} / a\right)^{1 / 2}>w^{1 / 2} \lambda^{-m / 2}\left(d_{1}-\alpha\right)^{-1 / 2} a^{-1 / 2}-\left(w 10 d_{2} \mu^{m} a^{-1}\right)^{1 / 2} .
$$

Combining, the ratio is greater than

$$
\begin{aligned}
\frac{w^{1 / 2} \lambda^{-m / 2}\left[a\left(d_{1}-\alpha\right)\right]^{-1 / 2}-w^{1 / 2} \mu^{m / 2}\left[10 d_{2} a^{-1}\right]^{1 / 2}}{w 10 d_{2} \mu^{m}+w^{1 / 2} \mu^{m / 2}\left[10 d_{2} a^{-1}\right]^{1 / 2}} & \geqq \frac{w^{1 / 2} \lambda^{-m / 2}\left[a d_{1}\right]^{-1 / 2} 2^{-1}}{w^{1 / 2} \mu^{m / 2} 4\left[d_{2} a^{-1}\right]^{1 / 2}} \\
& =(\mu \lambda)^{-m / 2} 8^{-1} d_{1}^{-1 / 2} d_{2}^{-1 / 2}
\end{aligned}
$$

Since $\mu \lambda<1$, this ratio can be made as large as desired for large $n$. The argument in [N4, pp. 134-136] shows this estimate implies the stable thickness $\tau^{s}\left(\Lambda_{n}(t)\right)$ is as large as desired for large $n$.

Proof of $7.2 d$. To show $\Lambda_{n}(t)$ has a hyperbolic structure, it is sufficient to find invariant unstable sectors $S(z) \subset T_{z} M$ for $z$ in $F_{t}^{n}\left(B_{n}\right) \cap B_{n}$ which are invariant and expanded by $D F_{t}^{k n}(z)$ for large $k,\left|D F_{t}^{k n}(z) v\right|>v$ for $v$ in $S(z)$. Unfortunately the vectors in the unstable sectors are not expanded for $k=1$, but there is a power $j=k(z) n$ depending on the point $z$ which expands vectors. By a compactness argument there is one power $k n$ which works for all points in $\Lambda_{n}(t)$ proving the existence of an expanding invariant subbundle. The existence of the stable bundle is similar and even slightly easier because $\mu<\lambda^{-1}$.

The first step is to define the sectors $S(z)$. For $z=(x, y)$ in $F_{t}^{n}\left(B_{n}\right) \cap B_{n}$ and $z_{-1}=F_{t}^{-n}(z)$, the slope of $\left(\partial F_{t}^{n} / \partial y\right)\left(z_{-1}\right)$ is about $2 a\left(x-x_{n}(t)\right)$. Define $\xi(z), \xi^{+}(z)$, and $\eta>1$ by

$$
\begin{gathered}
\xi(z)=2 a\left(x-x_{n}(t)\right), \\
\xi^{+}(z)=|\xi(z)|=2\left[a\left(y-y_{n}(t)\right)\right]^{1 / 2}, \\
1<\eta<2^{1 / 6}=\min \left\{2^{1 / 6}, 4 / 3,2^{3 / 4}\right\} .
\end{gathered}
$$


The sectors are defined by

$$
S(z)=\left\{v=\left(v_{1}, v_{2}\right) \in T_{z} M: \eta^{-1} \leqq v_{2} /\left[v_{1} \xi(z)\right] \leqq \eta\right\} .
$$

Thus for $v$ in $S(z)$ the slope of $v$ lies between $\eta^{-1} \xi(z)$ and $\eta \xi(z)$. This allows for either positive or negative slope depending on the sign of $\xi(z)$, i.e. the sign of $x-x_{n}(t)$.

7.5. Lemma. The sectors are invariant under $D F_{t}^{n}$, i.e. if $z$ and $F_{t}^{n}(z)$ are in $B_{n} \cap F_{t}^{n}\left(B_{n}\right)$, then $D F_{t}^{n}(z) S(z) \subset S\left(F_{t}^{n}(z)\right)$.

Proof. If $v=\left(v_{1}, v_{2}\right) \in S(z)$, then $\left(v_{1}^{\prime}, v_{2}^{\prime}\right) \equiv D F_{t}^{m}(z)\left(v_{1}, v_{2}\right)=\left(\mu^{m} v_{1}, \lambda^{m} v_{2}\right)$ is nearly vertical:

$$
\begin{aligned}
\left|v_{2}^{\prime} / v_{1}^{\prime}\right| & =\left|\lambda^{m} v_{2} / \mu^{m} v_{1}\right| \geqslant\left(\lambda \mu^{-1}\right)^{m} \eta^{-1} \xi^{+}(z) \\
& \geqslant\left(\lambda \mu^{-1}\right)^{m} \eta^{-1} 2(a g)^{1 / 2} \geqslant\left(\lambda \mu^{-1}\right)^{m} \eta^{-1} 2\left(\lambda \mu^{-1}\right)^{m-\alpha m} \\
& \geqslant \eta^{-1} 2\left(\lambda \mu^{-1}\right)^{\alpha m},
\end{aligned}
$$

which is arbitrarily large for large $n$ because $\lambda \mu^{-1}>1$. Then $v^{\prime \prime}=\left(v_{1}^{\prime \prime}, v_{2}^{\prime \prime}\right)$ $=D F_{t}^{n}(z)\left(v_{1}, v_{2}\right)=D H_{t}\left(F_{t}^{m}(z)\right)\left(v_{1}^{\prime}, v_{1}^{\prime}\right)$ has slope about $\xi\left(F_{t}^{n}(z)\right)$ which is between $\eta^{-1} \xi\left(F_{t}^{n}(z)\right)$ and $\eta \xi\left(F_{t}^{n}(z)\right)$. Thus $v^{\prime \prime}$ lies in $S\left(F_{t}^{n}(z)\right)$. This proves Lemma 7.5.

Next we show for $z$ bounded away from the bend at the bottom, the vectors in $S(z)$ are immediately expanded.

7.6. Lemma. If $z=(x, y)$ is in $B_{n}$ with $y-y_{n}(t)=\gamma$ h and $v$ is in $S(z)$, then $\left\|D F_{t}^{n}(z) v\right\|$ $\geqq 4 \gamma^{1 / 2} \eta^{-1}(1-\alpha)\|v\|$. If $\gamma \geqq 1 / 12$, then $\left\|D F_{t}^{n}(z) v\right\|>\|v\|$.

Proof. Let $v^{\prime \prime}=D F_{t}^{n}(z) v$. Then

$$
\begin{aligned}
\left\|v^{\prime \prime}\right\| & \geqslant\left(d_{1}-\alpha\right)\left\|v^{\prime}\right\| \geqslant d_{1}(1-\alpha)\left|v_{2}^{\prime}\right|=d_{1}(1-\alpha) \lambda^{m}\left|v_{2}\right| \\
& \geqslant d_{1}(1-\alpha) \lambda^{m} \eta^{-1} \xi^{+}(z)\left|v_{1}\right| \geqslant d_{1}(1-\alpha) \\
& \geqslant d_{1}(1-\alpha) \lambda^{m} \eta^{-1} \xi^{+}(z)\left[1+\eta^{2} \xi^{+}(z)^{2}\right]^{-1 / 2}\|v\| \\
& \geqslant d_{1}(1-\alpha) \lambda^{m} \eta^{-1} \xi^{+}(z)\|v\| \\
& \geqslant d_{1}(1-\alpha) \lambda^{m} \eta^{-1} 2[a \gamma h]^{1 / 2} \\
& \geqslant 4 \gamma^{1 / 2} \eta^{-1}(1-\alpha)\|v\| .
\end{aligned}
$$

For $\gamma \geqq 1 / 12,4 \gamma^{1 / 2} \eta^{-1}(1-\alpha)>1$ by the choice of $\eta$. This proves (7.6).

Even for the larger gap $g$ allowed in [N4], it is not possible for all points in $B_{n}$ to have $y-y_{n}(t) \geqq h / 12$. For $z$ with $y-y_{n}(t) \leqq h / 12$, the derivative $D F_{t}^{n}(z)$ contracts vectors in $S(z)$, but the next few iterates $z_{j}=F_{t}^{n j}(z)$ for $1 \leqq j \leqq k$ have $y_{j}-y_{n}(t) \geqq h / 2$, and so $D F_{t}^{n}\left(z_{j}\right)$ expands vectors in $S\left(z_{j}\right)$. Claim 7.7 below shows that the total effect of $D F_{t}^{n(k+1)}(z)=D F_{t}^{n}\left(z_{k}\right) \ldots D F_{t}^{n}\left(z_{1}\right) D F_{t}^{n}(z)$ is to expand vectors in $S(z)$. More precisely let $y_{0}$ be the value of $y$ on the bottom of $B_{n}$ and $H_{1}=\left\{z \in B_{n}: y-y_{0} \geqq h / 2\right\}$. For $z=(x, y)$ with $y-y_{0} \leqq h / 8$, let $k=k(z)$ be the integer such that $F_{t}^{n j}(z) \in H_{1}$ for $1 \leqq j$ $\leqq k(z)$ and $F_{t}^{n(k+1)}(z) \notin H_{1}$.

7.7. Lemma. If $y-y_{0} \leqq h / 12$, then for $k=k(z)$ as above and $v$ in $S(z)$

$$
\left\|D F_{t}^{n k+n}(z) v\right\| \geqq(1-\alpha) \eta^{-1} 2\|v\|>\|v\| \text {. }
$$


Proof. Let $z=(x, y)$ be in $B_{n}$ with $y-y_{0}=\gamma h$. To estimate the number of iterates $z$ stays in $H_{1}, k(z)$, we need to show $z_{1}=\left(x_{1}, y_{1}\right)=F_{t}^{n}(z)$ is very near the top of $B_{n}$. Also we need to compare the maximal and minimal stretches of vectors in $S(\zeta)$ for $\zeta$ in $H_{1}$.

The partial derivative $\left|\partial F_{t}^{n} / \partial y\right| \leqq(1+\alpha) d_{1} \lambda^{m}$. The slope of the image $F_{t}^{n}\left(B_{n}\right)$ is always less than the slope at the top, $s$,

$$
s \leqq 2\left[a\left(h+g^{\prime}\right)\right]^{1 / 2}<2 a^{1 / 2} h^{1 / 2}(1+\alpha)<4(1+\alpha) d_{1}^{-1} \lambda^{-m},
$$

because $g^{\prime} / h$ is small. Let $y_{\text {top }}$ be the value of $y$ on the top of $B_{n}$. Then

$$
y_{\text {top }}-y_{1} \leqq \gamma h(1+\alpha) d_{1} \lambda^{m} s \leqq \gamma h(1+\alpha) 4<h / 2,
$$

because $\gamma \leqq 1 / 12<1 / 8$. Thus $z_{1}$ is in $H_{1}$ and $y_{\text {top }}-y_{1}$ is proportional to $\gamma h$.

Let $\lambda_{\max }$ and $\lambda_{\max }$ be the maximal and minimal stretch of vectors in $S(\zeta)$ for $\zeta$ in $H_{1}$. Similarly let $\xi_{\max }$ and $\xi_{\max }$ be the maximal and minimal value of $\xi^{+}(\zeta)$ for $\zeta$ in $H_{1}$. Then

$$
\xi_{\max } \leqq 2\left[a h+a g^{\prime}\right]^{1 / 2} \leqq 2 a^{1 / 2} h^{1 / 2}(1+\alpha) \leqq 4 d_{1}^{-1} \lambda^{-m}(1+\alpha),
$$

and

$$
\xi_{\max } \geqq 2[a h / 2]^{1 / 2} \geqq 2^{1 / 2} a^{1 / 2} h^{1 / 2} \geqq 2^{1 / 2} 2 d_{1}^{-1} \lambda^{-m}
$$

Then

$$
\lambda_{\max } \leqq \lambda^{m}\left(d_{1}+\alpha\right) \eta \xi_{\max } \leqq 4 \eta(1+\alpha),
$$

and

$$
\lambda_{\min } \geqq \lambda^{m}\left(d_{1}-\alpha\right) \eta^{-1} \xi_{\min } \geqq 2^{1 / 2} 2 \eta^{-1}(1-\alpha) .
$$

By the coice of $\eta, \lambda_{\min } \geqq\left(\lambda_{\max }^{1 / 2}\right) 2^{1 / 4}$ and $\lambda_{\min }^{k} \geqq \lambda_{\max }^{k / 2} 2^{1 / 4}$ because $k(z) \geqq 1\left(z_{1}\right.$ is in $\left.H_{1}\right)$.

Using the fact $z_{1}$ is a distance at most $\gamma h(1+\alpha) d_{1} \lambda^{m}$ down the strip $F_{t}^{n}\left(B_{n}\right)$ and that $F_{t}^{n}$ stretches lengths along $F_{t}^{n}\left(B_{n}\right)$ at most $\lambda_{\max }$, it follws that the value $k=k(z)$ satisfies

$$
\begin{gathered}
\gamma h(1+\alpha) d_{1} \lambda^{m} \lambda_{\text {max }}^{k} s \geqq h / 2, \\
h \gamma 4(1+\alpha) \lambda_{\text {max }} \geqq h / 2, \\
\lambda_{\max }^{k} \geqq(1-\alpha) /(8 \gamma),
\end{gathered}
$$

and

$$
\lambda_{\max }^{k} \geqq(1-\alpha) /\left(\gamma^{1 / 2} 2^{5 / 4}\right) .
$$

Thus for $v$ in $S(z)$ and $y-y_{n}(t)=\gamma h \leqq h / 12$

$$
\begin{aligned}
\left\|D F_{t}^{n k+n}(z) v\right\| \cdot\|v\|^{-1} & \geqq \lambda^{m}\left(d_{1}-\alpha\right) \eta^{-1} \xi^{+}(z) \lambda_{\min }^{k} \\
& \geqq(1-\alpha) \lambda^{m} d_{1} \eta^{-1} 2[a \gamma h]^{1 / 2} \gamma^{-1 / 2} 2^{-5 / 4} \\
& \geqq(1-\alpha) \eta^{-1} 2^{3 / 4} \\
& >1
\end{aligned}
$$

by the choice of $\eta$. This completes the proof of (7.7). 
7.8. Proposition. For $g$ as given in (7.4), the maximal invariant set in $B_{n}$ for $F_{t}^{n}$, $\Lambda_{n}(t)$, has a hyperbolic structure.

Proof. The sector $S(z)$ is mapped inside the sector $S\left(F_{t}^{n}(z)\right)$ by Lemma 7.5. Therefore there is an invariant bundle $E_{z}^{u}$ for $z$ in $\Lambda_{n}(t)$. By Lemmas 7.6 and 7.7, the vectors $v$ in $E_{z}^{u}$ are eventually expanded. Since this is true for each $z$ in $\Lambda_{n}(t)$, there are $C>0$ and $\lambda_{u}>1$ such that for $v$ in $E_{z}^{u}$ and $j \geqq 0,\left\|D F_{t}^{n j}(z) v\right\| \geqq C \lambda_{u}^{j}\|v\|$. (This follows because all the Lyapunov characteristic exponents are positive.)

To show there is a contracting bundle, let $S^{*}(z)$ be the complementary sector to $S(z)$. It is overflowing by $D F_{t}^{n}(z)$ and so is invariant under $D F_{t}^{-n}(z): D F_{t}^{-n}(z) S^{*}(z)$ $C S^{*}\left(F_{t}^{-n}(z)\right)$. Therefore there is an invariant bundle $E_{z}^{s}$ for $z$ in $\Lambda_{n}(t)$. By the hypothesis that $\mu \lambda<1$, it follows that $\operatorname{det}\left(D F_{t}^{n}(z)\right)<1$. Since $D F_{t}^{n}(z)$ is expanding on the invariant bundle $E^{u}$, it has to be contracting on the complementary invariant bundle $E^{s}$. This completes the proof of Proposition 7.8 and (7.2d).

7.9. Remark. If the gap $g$ is smaller than allowed in (7.4) and is so small that a vector $v$ in a narrow sector $S(z)$ near the bottom of $B_{n}$ has an image $v^{\prime \prime}$ which is horizontal near the top of $B_{n}$, then $D F_{t}^{n}\left(z_{1}\right)$ would contract $v^{\prime \prime}$ again. Therefore for such a small gap, the maximal invariant set is probably not hyperbolic.

\section{Thick Stable Manifolds and Tangencies: Proof of Theorem D}

Proposition 7.1 proves that there are values $t=t_{n}^{*}$ when $F_{t}^{n}$ has a hyperbolic basic set $\Lambda_{n}\left(t_{n}^{*}\right) \subset B_{n}$ with $\tau^{s}\left(\Lambda_{n}\left(t_{n}^{*}\right)\right)$ arbitrarily large. There are also other values $t=t_{n}$, where $W^{u}\left(P_{t}, F_{t}\right)$ has a nondegenerate tangency with $W^{s}\left(P_{t}, F_{t}\right)$. The following result shows that there are parameter values where both phenomena occur simultaneously. Since being a wild hyperbolic set is open, Theorem D follows.

8.1. Proposition. For large enough $n$, there exists $t=t_{n}^{* *}$ for which (a) $F_{t}^{n}$ has a hyperbolic basic set $\Lambda_{n}^{\prime}\left(t_{n}^{* *}\right) \subset B_{n}$, (b) $F_{t}$ has a hyperbolic basic set $\Lambda\left(t_{n}^{* *}\right) \supset \Lambda_{n}^{\prime}\left(t_{n}^{* *}\right) \cup\left\{P_{t}\right\}$. (c) $\tau^{s}\left(\Lambda\left(t_{n}^{* *}\right) \tau^{u}\left(\Lambda\left(t_{n}^{* *}\right)\right)>1\right.$, and (d) $\Lambda\left(t_{n}^{* *}\right)$ has a persistent tangency of $W^{s}\left(\Lambda\left(t_{n}^{* *}\right)\right)$ and $W^{u}\left(\Lambda\left(t_{n}^{* *}\right)\right.$, i.e. $\Lambda\left(t_{n}^{* *}\right)$ is a wild hyperbolic set.

Proof. The idea of the proof is to pull back the parameter value from $t_{n}^{*}$ to $t=t_{n}^{* *}$ so that $F_{t}^{n}\left(B_{n}\right)$ does not come out the bottom of $B_{n}$ and there is a near tangency of $W^{s}\left(p_{n}\right)$ and $W^{u}\left(p_{n}\right)$, where $p_{n}=p_{n}(t)$ is a hyperbolic fixed point of $F_{t}^{n}$ in $B_{n}$. Done correctly, $p_{n}$ is still contained in a (smaller) hyperbolic basic set $\Lambda_{n}^{\prime}(t)$ with $\tau^{s}\left(\Lambda_{n}^{\prime}(t)\right)$ $\geqq \tau^{s}\left(\Lambda_{n}\left(t_{n}\right)\right)-\varepsilon$.

Before this is done we need a preliminary step. It is seen by the work of Silnikov that there is a parameter value $t=t_{1}$ near $t_{0}$ such that $W^{u}\left(P_{t}, F_{t}\right)$ has some point of transverse intersection with $W^{s}\left(P_{t}, F_{t}\right)$ and other points where there is a nondegenerate tangency. See [HG], [GS] or [N4]. See Fig. 11b. Thus we can assume that $P_{t}$ is already an element of a hyperbolic set $\Lambda_{1}\left(t_{1}\right)$.

Take $T$ such that $T \tau^{u}\left(\Lambda_{1}\left(t_{1}\right)\right)>4$. The thickness varies continuously for $C^{2}$ changes of $F_{t}$, [N4, Proposition 6.2], so there is an interval $I$ about $t_{1}$ such that for $t$ in $I, T \tau^{u}\left(\Lambda_{1}(t)\right)>2$. By the results of Sect. 7, for large enough $n$ there is a $t_{n}^{*}$ in $I$ and another hyperbolic set for $F_{t}^{n}, \Lambda_{n}\left(t_{n}^{*}\right) \subset B_{n}$, with $\tau^{s}\left(\Lambda_{n}\left(t_{n}^{*}\right)\right)>T$. There is a fixed point of $F_{t}^{n}$ in $\Lambda_{n}\left(t_{n}^{*}\right), p_{n}\left(t_{n}^{*}\right)$. Take $t=t_{n}^{* *}$ with $t_{1}<t_{n}^{* *}<t_{n}$ such that $F_{t}^{n}\left(B_{n}\right)$ does not 


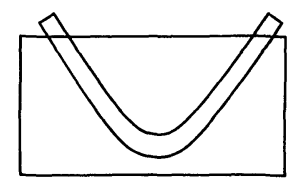

a)

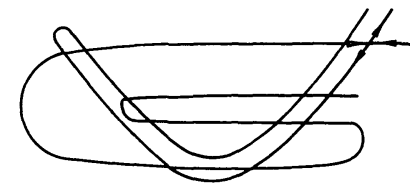

b)

Fig. 10 a. The image $F_{t}^{n}\left(B_{n}\right)$ is shown with $B_{n}$ for $t=t_{n}^{* *}: \mathbf{b}$ The position of the stable and unstable manifolds for $p_{n}$ are shown for $t=t_{n}^{* *}$

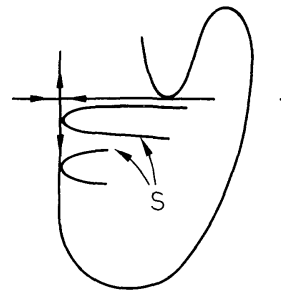

a)

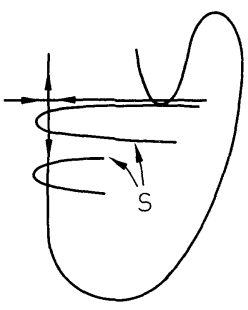

b)

Fig. 11a and b. The stable and unstable manifolds for $P_{t}$ are shown. The extra pieces of the stable manifold are labelled $S$. a $t=t_{0} ; \mathbf{b} t=t_{1}$

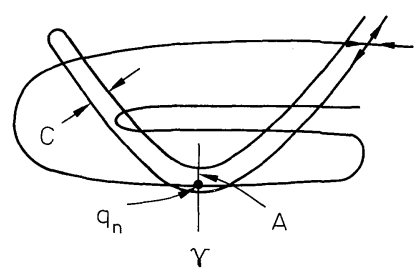

Fig. 12. " $A$ " labels the overlap of $\gamma \cap W^{s}\left(\Lambda_{n}^{\prime}\left(t_{n}^{* *}\right)\right)$ and $\gamma \cap W^{u}\left(\Lambda_{n}^{\prime}\left(t_{n}^{* *}\right)\right)$ which is of size about $w d_{2} \mu^{m} / 4$. The width " $C$ " between pieces of $W^{u}\left(p_{n}\left(t_{n}^{* *}\right)\right)$ is about $w d_{2} \mu^{m}$

come all the way out the bottom of $B_{n}$ and in fact $W^{u}\left(p_{n}(t), F_{t}^{n}\right)$ comes above but within $w d_{2} \mu^{m} / 4$ of a tangency with $W^{s}\left(p_{n}(t), F_{t}^{n}\right)$. See Fig. 12 . Note the first tangency of $W^{s}\left(p_{n}(s), F_{s}^{n}\right)$ and $W^{u}\left(p_{n}(s), F_{s}^{n}\right)$ occurs for $t_{n}^{* *}<s<t_{n}$, when there are still other points of transverse intersection of $W^{u}\left(p_{n}(s), F_{s}^{n}\right)$ and $W^{s}\left(p_{n}(s), F_{s}^{n}\right)$. We show below that there is a new smaller hyperbolic set for $F_{t}^{n}$ with $t=t_{n}^{* *}, \Lambda_{n}^{\prime}\left(t_{n}^{* *}\right)$, with

$$
\tau^{s}\left(\Lambda_{n}^{\prime}\left(t_{n}^{* *}\right)\right)>T,
$$

(8.2b) the Cantor set fo stable and unstable manifolds for $\Lambda_{n}^{\prime}\left(t_{n}^{* *}\right)$ overlap near the bottom of $F_{t}^{n}\left(B_{n}\right)$ for $t=t_{n}^{* *}$,

and

(8.2c) the stable and unstable manifolds of $\Lambda_{n}^{\prime}\left(t_{n}^{* *}\right)$ and $\Lambda_{1}\left(t_{n}^{* *}\right)$ intersect transversally. 


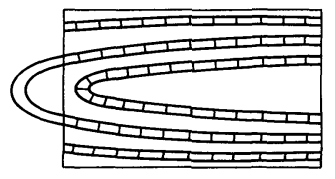

a)

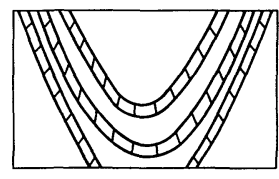

b)

Fig. 13. (a) The shaded region is $B(-J)$; (b) The shaded region is $B(J)$

Fig. 14. $U^{-}$is $F_{t}^{-n}(U)$

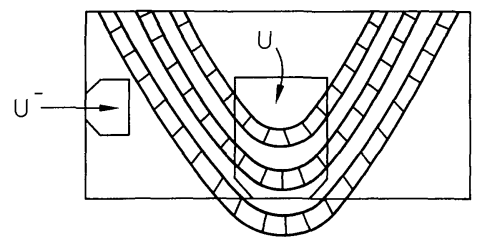

Using (8.2c), [N4, Lemma 8] proves there is a larger hyperbolic set $\Lambda\left(t_{n}^{* *}\right) \supset \Lambda_{1}\left(t_{n}^{* *}\right)$ $\cup \Lambda_{n}^{\prime}\left(t_{n}^{* *}\right)$. Then $\tau^{s}\left(\Lambda\left(t_{n}^{* *}\right)\right) \tau^{u}\left(\Lambda\left(t_{n}^{* *}\right)\right) \geqq \tau^{s}\left(\Lambda_{n}^{\prime}\left(t_{n}^{* *}\right)\right) \tau^{u}\left(\Lambda_{1}\left(t_{n}^{* *}\right)\right)>1$.

To use the thickness to show there is a persistent tangency, we need a $C^{1}$ curve $\gamma$ on which the minima of $W^{u}$ relative to $W^{s}$ occurs. Thus $\gamma$ is a curve of potential tangencies. If the manifolds $W^{s}\left(\Lambda\left(t_{n}^{* *}\right)\right)$ could be extended to a $C^{2}$ foliation, the existence of $\gamma$ would be easy: we could take $C^{2}$ coordinates such that these manifolds were straight horizontal lines and look at the minima of $W^{s}\left(\Lambda\left(t_{n}^{* *}\right)\right)$ in these coordinates. The argument [N4, Lemma 9 and pp. 119-120] modifies this argument so it works with only a foliation whith $C^{1}$ tangent vectors. The sketch of this proof is as follows. The Cantor set of unstable manifolds, $W^{s}\left(\Lambda\left(t_{n}^{* *}\right)\right)$ can be extended near $q_{n}$ [a point on $W^{s}\left(p_{n}\right)$ near the minimum of $W^{u}\left(p_{n}\right)$, see Fig. 12] to a foliation $\mathscr{F}^{u}$ with $C^{1}$ tangent vectors. Similarly $W^{s}\left(\Lambda\left(t_{n}^{* *}\right)\right)$ can be extended near $q_{n}$ to $\mathscr{F}^{s}$. The foliations $\mathscr{F}^{u}$ and $\mathscr{F}^{s}$ will have nondegenerate tangencies and so intersect along a $C^{1}$ curve $\gamma$. It then follows that for a small enough curve $\gamma_{\varepsilon}$ around $q_{n}, \tau\left(W^{s}\left(\Lambda\left(t_{n}^{* *}\right)\right) \cap \gamma_{\varepsilon}\right) \tau\left(W^{u}\left(\Lambda\left(t_{n}^{* *}\right)\right) \cap \gamma_{\varepsilon}\right)>1$, since the thickness can be measured along any $C^{1}$ curve. Therefore these two Cantor sets intersect and $W^{\mathrm{s}}\left(\Lambda\left(t_{n}^{* *}\right)\right)$ has a persistent tangency with $W^{u}\left(\Lambda\left(t_{n}^{* *}\right)\right)$ proving (8.1d).

Fix $t=t_{n}^{* *}$, which is chosen as above. We end this section by showing there is a hyperbolic basic set $\Lambda_{n}^{\prime}(t)$ for $F_{t}^{n}$ satisfying $(8.2 \mathrm{a}-\mathrm{c})$. This clarifies the last paragraph of the proof of [N4, Lemma 7] on p. 138.

Let $B(-J)=\bigcap_{j=0}^{-J} F_{t}^{n j}\left(B_{n}\right)$ and $B(J)=\bigcap_{j=0}^{J} F_{t}^{n j}\left(B_{n}\right)$. The set $B(J)$ is made up of "vertical" strips of width about $w d_{2}^{J} \mu^{m J}$ and $B(-J)$ of "horizontal" strips of width about $w C \lambda^{-m-m J}$. See Fig. 13.

The idea is to remove the part of $B_{n}$ near the bottom where the slope of $\partial F_{t}^{n} / \partial y$ is less than $\delta_{n}$, where $\delta_{n}=2\left[a w d_{2} \mu^{m} / 4\right]^{1 / 2} \eta^{-1}$. If any part of a component of $B(-J) \cap B(J)$ is removed, then the whole component should be removed. Let the set removed be denoted by $U$, and let $B_{n}^{\prime}=B_{n}-U$. Let $B^{\prime}(J)=\bigcap_{j=0}^{J} F_{t}^{n j}\left(B_{n}^{\prime}\right), B^{\prime}(-J)$ 
$=\bigcap_{j=0}^{-J} F_{t}^{n j}\left(B_{n}^{\prime}\right)$, and $B^{\prime}(-J, J)=B^{\prime}(-J) \cap B^{\prime}(J)$. Then $B_{n}^{\prime}(-J, J)$ is made up of boxes. In $B_{n}^{\prime}(-J, J), \partial F_{t} / \partial y$ has slope greater than $\delta_{n}$. Therefore the maximal invariant set in $B_{n}^{\prime}, A_{n}^{\prime}(t)=\bigcap_{j} F_{t}^{n j}\left(B_{n}^{\prime}\right)=\bigcap_{j} F_{t}^{n j} B_{n}^{\prime}(-J, J)$, has a hyperbolic structure by the proof of Proposition 7.8. [Note the tangencies in $F_{t}^{n}(U)$ are also removed.] For $J$ large enough, the height of the hook of a strip in $B(J)$ below the intersection with $B_{n}^{\prime}$ is less than $g^{\prime}=w 10 d_{2} \mu^{m}=h 10 d_{2}\left(d_{1}-\alpha\right)(\mu \lambda)^{m}$. The length left in $B_{n}^{\prime}$ is approximately as before. Therefore the argument as before shows that $\tau^{s}\left(\Lambda_{n}^{\prime}(t)>T\right.$, condition (8.2a). The Cantor sets of stable and unstable manifolds overlap because $p_{n}(t)$ is in $\Lambda_{n}^{\prime}(t)$ and the choice of $t_{n}^{* *}$, condition (8.2b).

Condition (8.2c) follows by looking at $F_{t}^{n}$ on $W^{u}\left(p_{n}\right)$. Each iterate extends a local unstable manifold further up until it crosses $W^{s}\left(P_{t}\right)$ transversally. See Figs. 6, 11 , and 12 . Similarly the manifolds $W^{s}\left(p_{n}\right)$ cut across $W^{u}\left(P_{t}\right)$ which cups around $\Lambda_{n}^{\prime}\left(t_{n}^{* *}\right)$. See Fig. 6 and [N4, p. 134]. This completes the proof of (8.2), Proposition 8.1 , and Theorem D.

8.3. Remark. For maps of the real line, $f(x)$, [or their corresponding graphs $F(x, y)$ $=(y, f(y))]$ it is also possible to create hyperbolic sets with $\tau^{s}$ as large as desired. However for maps of the interval $\tau^{u}=0$, so the product $\tau^{s} \tau^{u}=0$ is never greater than one. Therefore it is impossible to create persistent tangencies for maps of the interval. See [V] for a discussion of the comparison of maps of the interval and maps of the plane.

\section{Infinitely Many Sinks: Theorem E}

By the assumptions of the theorem, there is an interval of parameter values $J$ such that for each $t$ in $J$ there is a nondegenerate tangency of $W^{s}\left(\Lambda(t), F_{t}\right)$ and $W^{u}\left(\Lambda(t), F_{t}\right)$. Because $\Lambda(t)$ is a hyperbolic set with a dense orbit, the manifolds $W^{s}\left(P_{t}, F_{t}\right)$ and $W^{u}\left(P_{t}, F_{t}\right)$ are dense in $W^{s}\left(\Lambda(t), F_{t}\right)$ and $W^{u}\left(\Lambda(t), F_{t}\right)$, respectively. Because $W^{s}\left(\Lambda(t), F_{t}\right)$ and $W^{u}\left(\Lambda(t), F_{t}\right)$ have a tangency for each $t$ in $J$ and because this tangency changes location by assumption (iv) in the definition of nondegenerate creation of homoclinic intersection, it follows that there is a dense set of parameter values $J^{\prime} \subset J$ such that for $t$ in $J^{\prime}$ the manifolds $W^{s}\left(P_{t}, F_{t}\right)$ and $W^{u}\left(P_{t}, F_{t}\right)$ have nondegenerate tangencies. By Proposition 3.3 and the argument of Sect. 5, the set $J_{1} \subset J$ with at least one sink is dense. It is also open. Since $J^{\prime} \cap J_{1}$ is dense, repeating the argument proves that the set $J_{2}$ with at least two sinks is dense and open. By induction, the set $J_{k}$ with at least $k$ sinks is dense and open. Therefore $J_{\infty}=\bigcap\left\{J_{k}: k=1,2, \ldots\right\}$ is a residual subset of $J$ in the sense of Baire category. For $t$ in $J_{\infty}, F_{t}$ has infinitely many sinks. 


\section{References}

[ACHM] Aronson, D.G., Chory, M.A., Hall, G.R., McGehee, R.P.: Bifurcation from an invariant circle for two-parameter families of maps of the plane: a computer-assisted study. Commun. Math. Phys. 83, 303-354 (1982)

[CJ] Curry, J.H., Johnson, J.R.: On the rate of approach to homoclinic tangency. Preprint, University of Minnesota, 1982

[GS] Gavrilov, N.K., Silnikov, L.P.: On the three dimensional dynamical systems close to a system with a structurally unstable homoclinic curve. I. Math. USSR Sbornik 17, 467-485 (1972); II. Math. USSR Sbornik 19, 139-156 (1973)

[GH] Guckenheimer, J., Holmes, P.: Nonlınear oscillations, dynamical systems and bifurcation of vector fields. Applied Math. Sci. 42. Berlin, Heıdelberg, New York: Springer 1983 1983

[H] Hénon, M.: A two dimensional mapping with a strange attractor. Commun. Math. Phys. 50, 69-78 (1976)

[HP] Hirsch, M., Pugh, C.: Stable manifolds and hyperbolic sets. Proc. Am. Math. Soc. Symp. Pure Math. 14, 133-163 (1970)

[HM] Holmes, P., Marsden, J.: A partial differential equation with infinitely many periodic orbits. Archive Rational Mech. Anal. 76, 135-166 (1981)

[L] Levi, M.: Qualitative analysis of periodically forced relaxation oscillations. Memoirs Amer. Math. Soc. 32, 244 (1981)

[Mis] Misiurewicz, M.: Strange attractors for the Lozi mappings, nonlinear dynamics. Helleman, R. (ed.). Annals of New York Academy of Science 357 (1980)

[M] Moser, J.: Stable and random motions in dynamical systems. Ann. of Math. Studies 77, Princeton, NJ: Princeton University Press 1973

[N1] Newhouse, S.: Nondensity of axiom $A(a)$ on $S^{2}$. Proc. A.M.S. Symp. in Pure Math. 14, 191-203 (1970)

[N2] Newhouse, S.: Diffeomorphisms with infinitely many sinks. Topology 13, 9-18 (1974)

[N3] Newhouse, S.: Quasi-elliptic periodic points in conservative dynamical systems. Am. J. Math. 99, 1061-1087 (1977)

[N4] Newhouse, S.: The abundance of wild hyperbolic sets and non-smooth stable sets for diffeomorphisms. Publ. Math. IHES 50, 101-151 (1979)

[N5] Newhouse, S.: Lectures on dynamical systems. Progress in Math. 8, 1-114. Boston: Birkhäuser 1980

[V] Van Strien, S.: On the bifurcations creating horseshoes. Lecture Notes in Mathematics, Vol. 898. Rand, D., Young, L.-S. (eds.), pp. 316-351. Berlin, Heidelberg. New York: Sprınger 1981

Communicated by O. E. Lanford

Received February 7, 1983; in revised form May 16, 1983 
